\title{
Beyond the mini-solar maximum of solar cycle 24: Declining solar magnetic fields and the response of the terrestrial magnetosphere
}

\author{
M. Ingale ${ }^{1}$, P. Janardhan ${ }^{1}$, and S. K. Bisoi ${ }^{2}$ \\ ${ }^{1}$ Physical Research Laboratory, Astronomy \& Astrophysics Division, Navarangpura, Ahmedabad - 380 \\ 009, India. \\ ${ }^{2}$ Key Laboratory of Solar Activity, National Astronomical Observatories, Chinese Academy of Sciences, \\ Beijing 100012, China
}

\section{Key Points:}

- Global response of the terrestrial magnetosphere to the long term decline in solar magnetic fields

- Steady increase of terrestrial magnetopause and bow shock stand-off distance since around mid-1990's

- Forecast of an expanded terrestrial magnetopause during the minimum of present solar cycle 24 


\begin{abstract}
The present study examines the response of the terrestrial magnetosphere to the longterm steady declining trends observed in solar magnetic fields and solar wind micro-turbulence levels since mid-1990's that has been continuing beyond the mini- solar maximum of cycle 24. A detailed analysis of the response of the terrestrial magnetosphere has been carried out by studying the extent and shape of the Earth's magnetopause and bow shock over the past four solar cycles. We estimate sub-solar stand-off distance of the magnetopause and bow shock, and the shape of the magnetopause using numerical as well as empirical models. The computed magnetopause and bow shock stand-off distances have been found to be increasing steadily since around mid-1990's, consistent with the steady declining trend seen in solar magnetic fields and solar wind micro-turbulence levels. Similarly, we find an expansion in the shape of the magnetopause since 1996. The implications of the increasing trend seen in the magnetopause and bow shock stand-off distances are discussed and a forecast of the shape of the magnetopause in 2020, the minimum of cycle 24, has been made. Importantly, we also find two instances between 1968 and 1991 when the magnetopause stand-off distance dropped to values close to 6.6 earth radii, the geostationary orbit, for duration ranging from 9-11 hours and one event in 2005, post 1995 when the decline in photospheric fields began. Though there have been no such events since 2005, it represents a clear and present danger to our satellite systems.
\end{abstract}

\title{
1 Introduction
}

The long term variability in solar magnetic fields can induce changes in the terrestrial magnetosphere, with the solar wind providing the complex link through which the effect is mediated. This link has been of particular interest to the solar and space science community due to the peculiar behaviour seen in solar cycles 23 and 24 and in view of the long term changes taking place on the sun and in the solar wind over the past three solar cycles. The variation of photospheric fields obtained from ground-based magnetic field measurements since 1970's have been used as an indicator of solar cycle activity. Our studies of solar photospheric magnetic fields (Bisoi, Janardhan, Chakrabarty, Ananthakrishnan, \& Divekar, 2014; Janardhan et al., 2015; Janardhan, Bisoi, \& Gosain, 2010; Janardhan, Fujiki, Ingale, Bisoi, \& Rout, 2018) in the past few years have shown a steady and continuous decline of solar high-latitude photospheric fields since mid-1990's. In earlier studies, we have also shown the steady decline in solar wind micro-turbulence levels in the inner heliosphere, spanning heliocentric distances from 0.2 to $0.8 \mathrm{AU}$ (Bisoi, Janardhan, Ingale, et al., 2014; Janardhan, Bisoi, Ananthakrishnan, Tokumaru, \& Fujiki, 2011; Janardhan et al., 2015), in sync with the decline in photospheric magnetic fields. The solar wind turbulence levels were obtained using ground based interplanetary scintillation (IPS) measurements. IPS essentially provides one with an idea of the large scale structure of the solar wind (Ananthakrishnan, Balasubramanian, \& Janardhan, 1995; Ananthakrishnan, Coles, \& Kaufman, 1980). Early, IPS measurements however, were primarily employed in determining angular sizes of radio sources (Janardhan \& Alurkar, 1993; Readhead \& Hewish, 1972). More recent observations have provided deep insights into the global structure of the solar wind and heliospheric magnetic field (HMF) all the way out to the solar wind termination shock at $~ 90 \mathrm{AU}$, where $1 \mathrm{AU}$ is the sun-earth distance (Fujiki, Tokumaru, Hayashi, Satonaka, \& Hakamada, 2016). The long term declining trends seen in both photospheric magnetic fields and solar wind micro-turbulence levels over the entire inner-heliosphere, coupled with the unusually deep solar minimum in cycle 23 and the very unusual solar polar field conditions seen in cycle 24 (Gopalswamy, Yashiro, \& Akiyama, 2016; Janardhan et al., 2018), implies that these changes could directly affect the terrestrial magnetosphere.

One way to quantify the effect of the long term changes in solar cycle activity on the terrestrial magnetosphere (MS) is by investigating the size and shape of the bow shock (BS) and magnetopause (MP) under different solar wind conditions. The BS forms in 
the upstream region of the Earth's magnetosphere, followed by the magnetosheath which in turn is bounded on the earthward side by the MP. The changing shape and position of the BS and MP with time is important in understanding space weather and also in planetary exploration because much like the earth, other planetary magnetosphere would have also undergone changes in their MP shape as a result of the observed global changes occurring in the solar wind. Using empirical and numerical models, the location and shape of the MP and BS under various solar wind conditions have been predicted since a long time. Most of the empirical models (Boardsen, Eastman, Sotirelis, \& Green, 2000; Fairfield, 1971; Formisano, 1979; Jelínek, Němeček, \& Šafránková, 2012; Lin, Zhang, Liu, Wang, \& Gong, 2010; Nemecek \& Safrankova, 1991; Shue et al., 1997, 1998; Sibeck, Lopez, \& Roelof, 1991), with a few exceptions (Shukhtina \& Gordeev, 2015; Wang et al., 2013), assume a priori functional form for the MP and then estimate the free parameters using available spacecraft crossing database. For example, Shue et al. (1998) proposed the following functional form,

$$
r=r_{m p}\left(\frac{2}{1+\cos \theta}\right)^{\alpha} R_{E} .
$$

Equation (1) has two parameters viz. $\mathrm{r}_{m p}$, the MP stand-off distance and $\alpha$, the flaring parameter. Angle $\theta$ is the solar zenith angle (the angle between the Sun-Earth line and the radial direction) of the point of interest. This assumption can, however, introduce errors in the cusp regions of Earth's magnetosphere due to the asymmetric MP shape at that location. On the other hand, most of the numerical models used to determine the MP position and shape (Cairns \& Lyon, 1995; J. F. Chapman \& Cairns, 2003; Elsen \& Winglee, 1997; GarcíA \& Hughes, 2007; Peredo, Slavin, Mazur, \& Curtis, 1995), were based on non-axis symmetric global MHD simulations. So, unlike empirical models, the condition of pressure balance between the solar wind dynamic pressure and the pressure due to the earth's dipole magnetic field (Beard, 1960; S. Chapman \& Ferraro, 1931; Mead \& Beard, 1964; Olson, 1969; Spreiter \& Briggs, 1962; Zhigulevsk \& Romishevskii, 1959) is satisfied at every point. However, the numerical models generally don't include all magnetospheric current systems and often use an impermeable, infinitely conducting MP as an obstacle, which is far from reality. The BS shape and location is usually determined by the shape and location of MP as well as by various solar wind conditions, like for example the solar wind ram pressure, the magnetosonic and Alfvén Mach numbers and the orientation of the interplanetary magnetic field (IMF). Several empirical (Fairfield, 1971; Fairfield et al., 2001; Farris \& Russell, 1994; Formisano, 1979; Jelínek et al., 2012; Peredo et al., 1995) and numerical models (Cairns \& Grabbe, 1994; Cairns \& Lyon, 1995, 1996; J. F. Chapman \& Cairns, 2003) have been used to estimate the shape and location of the BS.

The actual shape or position for the BS and MP is however still far from reality. We have therefore used both empirical and numerical models together to study the position and shape of MP and BS by determining the stand-off distances of MP and BS as a function of time over the last four decades, 1975 - 2017, and have in turn linked them to the long-term trends in solar magnetic activity. It is to be noted however, that our study concentrates only on estimating the long-term trends in the MP and BS standoff distances and the shape of the MP. Therefore, in this paper, we estimate the MP standoff distance using models by Lin et al. (2010), and Lu et al. (2011), as being representative of empirical and numerical models, respectively see the model description in §3.1). Similarly, the bow shock and it's stand-off distance has been estimated using Jelínek et al. (2012) and J. F. Chapman and Cairns (2003) as being representative of empirical and numerical models, respectively see the model description in $§ 3.2$ ). The other study that we found for such long-term trends for the MP stand-off distances is by McComas et al. (2013), wherein, the authors reported the canonical stand-off distance of the MP to be about 11 earth radii $\left(R_{E}\right)$, for the period 2009 to 2013 , covering the minimum of cycle 23 to the early rise phase of cycle 24 , compared to about $10 \mathrm{R}_{E}$ for the period 1974 to 1994 , covering cycles $21-22$. While the normal value of the MP is usually $\sim 10 \mathrm{R}_{E}$. Hence, it is evident that the long term study of the shape and location of the BS and MP is im- 


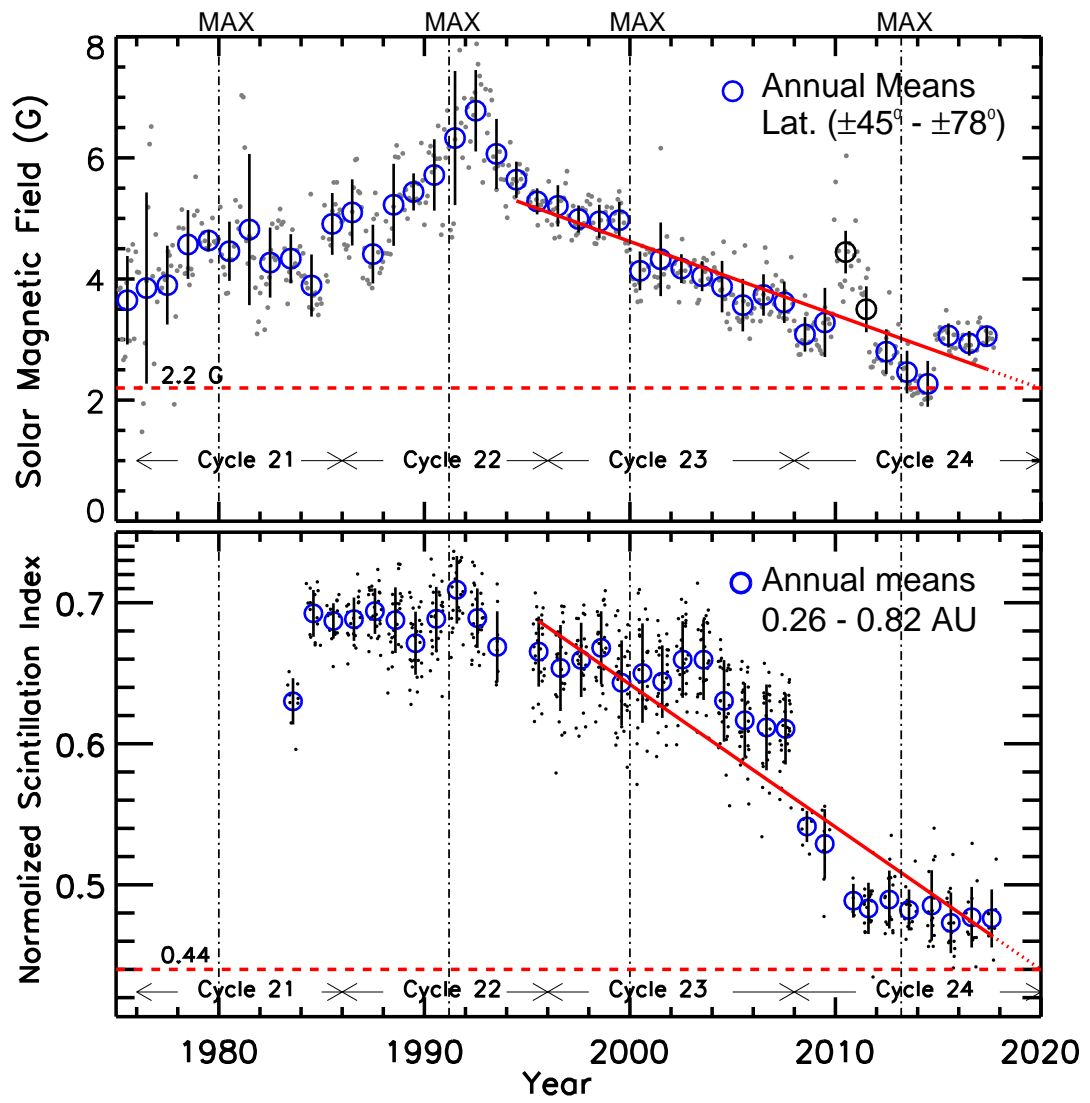

Figure 1. Solar high-latitude photospheric magnetic fields in the latitude range $45^{\circ} \quad-\quad-78^{\circ}$ for the period Feb. 1975-Dec. 2017 (upper panel) and solar wind micro-turbulence levels from $327 \mathrm{MHz}$ IPS observations in the period 1983-2017 and in the distance range 0.2-0.8 AU (lower panel). The filled grey dots in both panels are actual measurements of magnetic fields (top) and solar wind micro-turbulence (bottom), while the open blue circles are annual means shown with $1 \sigma$ error bars. The solid red line in both panels is a best fit to the declining trends for the annual means while the dotted red lines are extrapolations of the best fit until 2020 for the photospheric fields (top) and the IPS observations (bottom). The horizontal red dashed lines are marked at 2.2 $\mathrm{G}$ and 0.44 in the upper and lower panels, respectively. 
portant. Recently, Samsonov et al. (2019), using solar wind observations and empirical magnetopause models, also reported an increase in average annual magnetopause standoff distance by nearly $2 \mathrm{R}_{E}$ between $1991\left(9.7 \mathrm{R}_{E}\right)$ and $2009\left(11.6 \mathrm{R}_{E}\right)$. In this study we therefore, attempt to quantify the changes in shape and location of the Earth's magnetosphere over the last four solar cycles, and to compare the results obtained using both empirical and numerical models.

\section{Observations}

\subsection{Solar photospheric magnetic fields}

Figure 1 (upper panel) shows solar photospheric magnetic fields in the latitude range $45^{\circ}-78^{\circ}$ for the period Feb.1975-Dec.2017. Here, we selected the latitude range $45^{\circ}-78^{\circ}$ to represent the high-latitude solar polar fields. It must however, be kept in mind that different researchers have used different latitude ranges to estimate polar fields viz. poleward of $45^{\circ}$ (Bisoi, Janardhan, Chakrabarty, et al., 2014; Janardhan et al., 2011), 55 (Wilcox Solar Observatory polar fields, http://wso.stanford.edu/Polar.html, Janardhan et al. (2018)), 60 (de Toma, 2011; Gopalswamy et al., 2016, 2012; Sun, Hoeksema, Liu, \& Zhao, 2015), and 70 (Muñoz-Jaramillo, Sheeley, Zhang, \& DeLuca, 2012). The other point to note here that the polar fields studied by different researchers are actually the signed values of solar polar fields (see Figure 1 in Janardhan et al. (2018)), which reverse polarity around solar maximum of each solar cycle. However, in the present study, we emphasize only on the unsigned or absolute values of solar polar fields in the latitude range $45^{\circ}-78^{\circ}$. It is seen (see Figure 3 in Janardhan et al. (2015) and Figure 5 in Janardhan et al. (2018)) that the unsigned values of solar magnetic fields in the latitude range $0^{\circ}-45^{\circ}$ follow the solar cycle with the fields attaining their maximum at around solar maximum of each solar cycle.

The filled grey dots in the upper panel of Fig.1 are unsigned or absolute values of magnetic field measurements for each Carrington-rotation (CR) while the open blue circles are annual means, of Carrington rotation averaged magnetic fields, shown with $1 \sigma$ error bars. The unsigned Carrington-rotation averaged solar magnetic fields were computed using synoptic magnetograms from CR1625-CR2197, available as standard FITS format files, obtained from the National Solar Observatory, Kitt Peak (NSO/KP) and the Synoptic Optical Long-term Investigations of the Sun (NSO/SOLIS) facility. Each synoptic magnetograms represents arrays of $360 \times 180$ in longitude and sine of latitude format with magnetic field distribution for each Carrington rotation or 27.2753 day averaged photospheric magnetic fields in units of Gauss. Details about the computation of magnetic fields can be referred to in Janardhan et al. (2010, 2018).

It is evident from Fig.1 (upper panel) that the decline in the field strength of high latitude photospheric magnetic fields that has begun since $\sim 1995$ has been continuing till the end of Dec. 2017. However, for the years 2010-2011, a sudden increase in the field strength is first observed, while a similar increase is then observed for the years 20152017. It is to be noted however, that the decreasing trend in the field strength still persists. The 25-year long decreasing trend seen in the high-latitude field strength shows an $\sim 40 \%$ drop from its peak value in 1995 to the value in 2017. In Fig.1 (upper panel), the solid red line is a best fit, with a Pearson correlation coefficient of $r=-0.91$ at a significance level of $99 \%$, to the declining trend of high-latitude field strength for all the annual means in the period 1995-2017. Based on magnetic field measurements from Feb. 1975 - Jul. 2014, Janardhan et al. (2015) reported that the trend of declining high-latitude field strength would continue in the same manner up to the minimum of the current solar cycle 24 , i.e. $\sim 2020$. In fact, as evident from Fig.1, it is seen that the declining trend in the high latitude fields have been continuing. Thus, we expect the trend to continue in the same manner and extrapolate the solid red line beyond 2017 upto 2020, the expected minimum of the current solar cycle 24 as shown by the dotted red line, in Fig.1. 
The expected value of high-latitude field strength in 2020 is $\sim 2.2( \pm 0.08) \mathrm{G}$, as indicated in the upper panel of Fig. 1 by a dashed red horizontal line. A clear long term decreasing trend in solar high-latitude field strength is apparent and it is reasonable to assume that this trend will continue at least until 2020, the expected minimum of the current cycle.

\subsection{Solar wind micro-turbulence levels}

The lower panel in Fig.1 shows the solar wind micro-turbulence levels as measured by $327 \mathrm{MHz}$ IPS observations for the period 1983 to the end of 2017 and in the heliocentric distance range $0.2-0.8 \mathrm{AU}$, covering the inner heliosphere. These measurements were made using the three station IPS observatory of the Institute for Space-Earth Environmental research (ISEE), Nagoya, Japan. The measurements of scintillation index obtained from ISEE usually vary with the heliocentric distance and with the angular diameter of the observed radio source. As a result, the scintillation index measurements for a selected number of compact, point-like extra-galactic radio sources in the period 1983-2017 were normalized in a manner to make them independent of heliocentric distance and angular source size such that they should show a scintillation index of unity at a radial distance of $0.2 \mathrm{AU}$ (for more details see Janardhan et al. (2011) and Bisoi, Janardhan, Ingale, et al. (2014)). The filled grey dots in the lower panel of Fig. 1 are measurements of normalized scintillation index while the open blue circles are annual means shown with $1 \sigma$ error bars. The solid red line is a least square fit to the declining trend for the annual means with a Pearson correlation coefficient of $r=-0.93$, at a significance level of $99 \%$.

It is seen from Fig.1 (lower panel) that the normalized scintillation level has been steadily declining, in sync with the declining photospheric fields, until the end of 2017. The implication of the declining scintillation index is that a strongly scintillating pointlike, extra- galactic radio source at $327 \mathrm{MHz}$ would appear to scintillate like a much weaker and extended source. This is due to the significant decrease in the rms electron density fluctuations $\Delta \mathrm{N}$ in the solar wind over time. The fit to the normalized scintillation index values has been extrapolated beyond 2017 and is shown by a dotted red line in the lower panel of Fig.1. If the decline continues, the normalized scintillation index will reach a value of 0.44 by 2020, indicated by a horizontal red line in the lower panel of Fig.1. This is equivalent to IPS observations of an extra-galactic source with an angular diameter of $\sim 190$ milliseconds (mas). So a point source like 1148-001, with a measured angular size, using VLBI observations, of 10 mas will scintillate like a 190 mas source by 2020.

\subsection{Solar wind parameters at $1 \mathrm{AU}$}

The eight panels in Figure 2 show Carrington-rotation (or 27.2753 day) averaged solar wind parameters at 1 AU. Starting from the top and going down, the eight panels show respectively, the north-south component of IMF $\left(\mathrm{B}_{z}\right)$ in GSM coordinate system, the strength of IMF $(\mathrm{B})$, flow speed $(\mathrm{v})$, proton density $\left(\mathrm{N}_{p}\right)$, proton temperature $\left(\mathrm{T}_{p}\right)$, dynamic pressure $\left(\mathrm{P}_{d}\right)$ and alpha-to-proton number density ratio $\left(\mathrm{He}^{++} / \mathrm{H}^{+}\right)$and the Carrington-rotation averaged sunspot number $(\mathrm{R})$. The data shown span the period Feb. 1975-Dec. 2017, covering solar cycles 21-24. The gray vertical bands in Fig.2 indicate 1 year intervals during solar minima of cycles 20-23 corresponding to CR16421654, CR1771-1783, CR1905-1917, and CR2072-2084, respectively (Janardhan et al., 2015). The Carrington-rotation averaged solar wind parameters at 1 AU presented in Fig.2 were deduced from daily measurements obtained from the OMNI data base http://gsfc.nasa .gov/omniweb while the Carrington-rotation averaged sunspot number were derived from daily measurements of sunspot number obtained from the Royal Observatory Belgium, Brussels http://www.sidc.be/silso/datafiles. The Carrington-rotation averaged values were derived in order to compare them with the Carrington-rotation averaged photospheric magnetic fields as shown in Fig.1 (upper panel). The values of $\mathrm{B}, \mathrm{P}_{d}$ and $\mathrm{He}^{++} / \mathrm{H}^{+}$ 


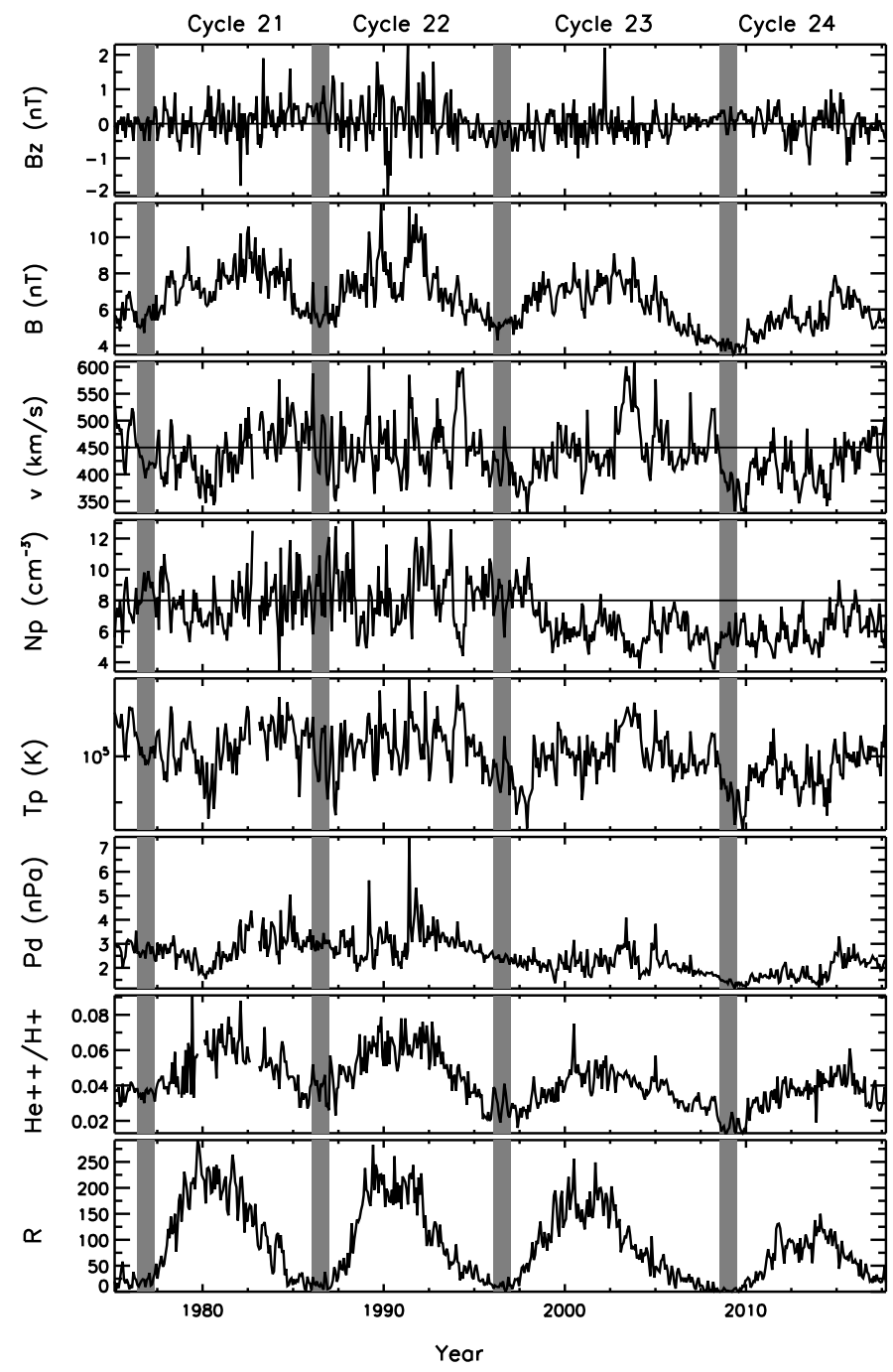

Figure 2. Carrington rotation-averaged insitu measurements of solar wind parameters at 1 $\mathrm{AU}$ (first to eighth panels) $\mathrm{B}_{z}, \mathrm{~B}, \mathrm{v}, \mathrm{N}_{p}, \mathrm{~T}_{p}, \mathrm{P}_{d}, \mathrm{He}^{++} / \mathrm{H}^{+}$, and $\mathrm{R}$, respectively, for the period Feb. 1975-Dec. 2017, covering solar cycles 21-24. The vertical gray bars demarcate 1 year intervals during the minima of solar cycles 20-23. The solid horizontal lines in third and fourth panels are drawn to indicate the average values of $\mathrm{v}$ and $\mathrm{N}_{p}$, respectively. 
in Fig.2 show a 11-year temporal variation. However, the temporal variation is not a oneto-one correspondence with sunspot activity variation. The important point to note from temporal variations of $\mathrm{B}, \mathrm{N}_{p}, \mathrm{v}, \mathrm{P}_{d}$ and $\mathrm{He}^{++} / \mathrm{H}^{+}$over the last four cycles is a long term decreasing trend that has begun around the mid-1990's and has been continuing till date. The value of $\mathrm{B}$ shows a decrease from $\sim 7.8 \mathrm{nT}$ in 1995 to $\sim 5.2 \mathrm{nT}$ in 2017, with an average decline of $\sim 30 \%$. The solar wind speed is also very low during cycle 24 with speeds being less than $\sim 450 \mathrm{~km} / \mathrm{s}$, most of the time as indicated by a horizontal line in the third panel of Fig.2. Not only the solar wind speed, but also we also see a significant reduction in solar wind proton density, especially starting from cycle 23 and continuing in current cycle 24 until the present. For most of the time, the value of proton density remained below $\sim 8 \mathrm{~cm}^{-3}$, indicated by a horizontal line in the fourth panel of Fig.2. While the average decline in $\mathrm{P}_{d}$, over the last $\sim 20$ years, was found to be $\sim 40 \%$. McComas et al. (2013) reported the weakest solar wind conditions during the maximum of current cycle 24 (their data spanned the period up to the maximum of cycle 24) and referred to the cycle 24 maximum as mini-solar maximum. Although, as seen from SSN in the eight panel of Fig.2, we are approaching the minimum of cycle 24, however, the weak nature of the solar wind as a whole has been still continuing. In addition, it is to be noted that the values of all solar wind parameters have remained at an all time low during the extended solar minimum of cycle 23. The long term decreasing trend of solar wind parameters at $1 \mathrm{AU}$ is in correspondence with the diminishing sunspot cycle activity seen since cycle 22 . The changes in solar wind conditions at $1 \mathrm{AU}$ observed in the recent period is in keeping with the declining photospheric magnetic fields and solar wind micro-turbulence levels in the inner heliosphere that began around the same period.


Figure 3. $\mathrm{B}$ (left panel) and $\mathrm{P}_{d}$ (right panel) as function of $\mathrm{B}_{p}$ for the period Feb. 1975-Dec. 2017. The respective Pearson's correlation coefficients of $r=0.40$ and 0.43 with confidence intervals of $99 \%$ are indicated in the top right corners of the panels. The filled black and red dots are measurements for the period Feb. 1975-Dec.1994 and Jan. 1995-Dec. 2017, respectively while the solid black lines in the panels are best fits to the all of the data points (both black and red dots).

In order to show a causal link of $\mathrm{B}$ and $\mathrm{P}_{d}$ with solar high-latitude fields $\left(\mathrm{B}_{p}\right)$, we plotted the correlation of $\mathrm{B}$ with $\mathrm{B}_{p}$ in the left panel of Figure 3 and that of $\mathrm{P}_{d}$ with $\mathrm{B}_{p}$ in the right panel of Fig.3. The solid black and red dots shown in Fig.3 are, respectively, the Carrington-rotation (27.2753 days) averaged data points for the period 1975-Dec. 1994 and Jan. 1995-Dec. 2017. It is to be remembered here that $\mathrm{B}_{p}$ have shown a steady 
decline since around 1995. The data points are, therefore, shown in solid black and red dots so as to compare the correlation of $\mathrm{B}$ and $\mathrm{P}_{d}$ with $\mathrm{B}_{p}$, prior to 1995 and after 1995. It can be seen from Fig.3 (left panel) that B show a correlation with $\mathrm{B}_{p}$ with a Pearson correlation coefficients of $r=0.40$ at a significance level of $99 \%$, indicated at the top right corner of the left panel of Fig.3. Similarly, a correlation between $\mathrm{P}_{d}$ and $\mathrm{B}_{p}$ with a correlation coefficient of $r=0.43$ at a significance level of $99 \%$ can be seen from Fig.3 (right panel). It is seen, as shown by the red dots, that post-1995 the values of B and $\mathrm{P}_{d}$ have decreased significantly. The solid black line in each panel in Fig.3 is a best fit to all data points (both black and red dots) of $\mathrm{B}$ and $\mathrm{P}_{d}$ with $\mathrm{B}_{p}$. The significant decrease in values of $\mathrm{B}$ and $\mathrm{P}_{d}$ post mid-1990's can be attributed to the weaker solar wind conditions in the inner heliosphere. The signatures of this weakening have already been shown in the solar wind turbulence levels in the inner heliosphere, as shown in Fig.1 (lower panel). Janardhan et al. (2015) reported a good correlation between $\mathrm{B}$ and $\mathrm{B}_{p}$ at solar minima using measurements for cycles 20-23. Here, we have revisited the correlation for $\mathrm{B}$ and $\mathrm{B}_{p}$ at solar minima, using Carrington-rotation averaged data for 1 year intervals at each solar minimum of cycles 20-23, corresponding to the Carrington-rotation period of CR1642-1654, CR1771-1783, CR1905-1917, and CR2072-2084, respectively. The correlation of $\mathrm{B}$ and $\mathrm{B}_{p}$ during solar cycle minima is plotted in the left panel of Fig.4. Similarly, the correlation of $\mathrm{P}_{d}$ with $\mathrm{B}_{p}$ during solar cycle minima is plotted in the right panel of Fig.4. The respective Person's correlation coefficients, $\mathrm{r}=0.50$ and 0.62 , are indicated in the top right corner of each panel. It is evident from the correlation
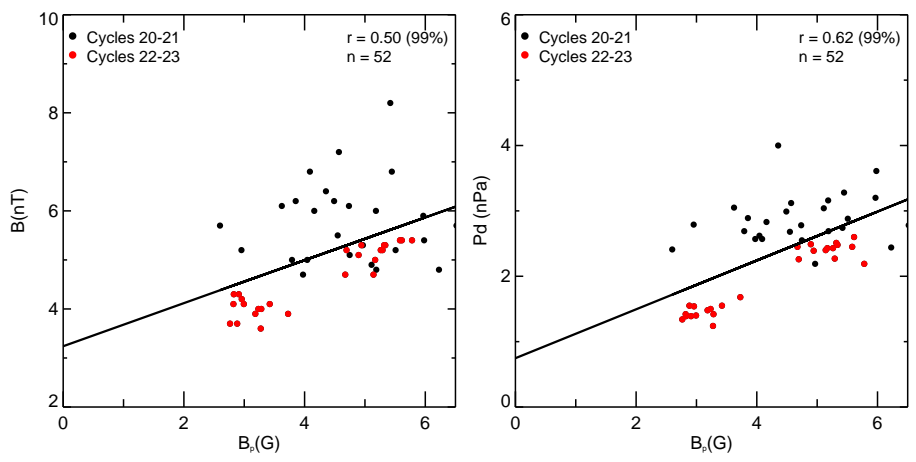

Figure 4. $\mathrm{B}$ (left panel) and $\mathrm{P}_{d}$ (right panel) as function of $\mathrm{B}_{p}$ for one year interval period during the minima of cycles 20-23. The respective Pearsons correlation coefficients, $\mathrm{r}=0.50$ and 0.62 , with confidence intervals are indicated in the top right corner of each panel, respectively. The filled black and red dots in each panel are measurements for cycles 20-21 and cycles 22-23, respectively. The solid black lines are best fit to all of the data points (both black and red dots).

coefficient values that $\mathrm{B}$ and $\mathrm{P}_{d}$ show a good correlation with $\mathrm{B}_{p}$. While in order to show the global reduction of $\mathrm{B}$ and $\mathrm{P}_{d}$ post mid-1990's, we plotted the data points for cycles 20-21 and cycles 22-23 using the filled black and red dots, respectively. It can be seen that post mid-1990's, i.e., for cycles 22 and 23, as shown by the red dots in each panel, we see a significant decrease in the strength of $\mathrm{B}$ and $\mathrm{P}_{d}$. Also, we found better correlations for $\mathrm{B}$ and $\mathrm{P}_{d}$ with $\mathrm{B}_{p}$ for data points covering the minima of cycles 22 and 23 with correlation coefficients of $\mathrm{r}=0.92$ and 0.95 , respectively. From Figures 2, 3 and 4, it is clear that the values of $\mathrm{B}$ and $\mathrm{P}_{d}$ have shown significant reductions during cycles 22-24, i.e., since around mid-1990's when solar high-latitude fields started to decline. As 
mentioned earlier, the location (or subsolar stand-off distance) of BS and MP depend on strength of $\mathrm{P}_{d}$ which show good correlations with solar polar fields, it is therefore important to know how the subsolar stand-off distances of BS and MP are related to the long term declining trend seen in solar polar fields since mid-1990's. In the following sections, we discuss the estimation of stand-off distances of BS and MP, and their temporal variation in keeping with the changes in solar polar fields.

\section{Methodology}

Figure 5 shows a schematic representation (not to scale) of the position and shape of the MP and BS in the GSM coordinate system wherein, the earth is considered to be at the origin. The $\mathrm{x}$-axis is along the sun-earth line, the $\mathrm{z}$-axis is perpendicular to the plane of the earth's orbit. $R_{\mathrm{bs}}$ and $R_{\mathrm{mp}}$ represent stand-off distances of the BS and MP, respectively. The nominal positions of the stand-off distances of the MP and BS at 10 and 14 earth radii $\left(\mathrm{R}_{E}\right)$ respectively, are indicated. Also shown, by a red circle at 6.6 $\mathrm{R}_{E}$, is the geostationary orbit in the earth's equatorial plane and the location of the Lagrangian point, L1, of the sun-earth system at $232 \mathrm{R}_{E}$.

\subsection{MP stand-off distance}

We briefly describe below the models used in the calculations of the MP stand-off distance and MP shape. The first model is by Lin et al. (2010) which represents an empirical approach, whereas the second model by Lu et al. (2011) is representative of a numerical approach.

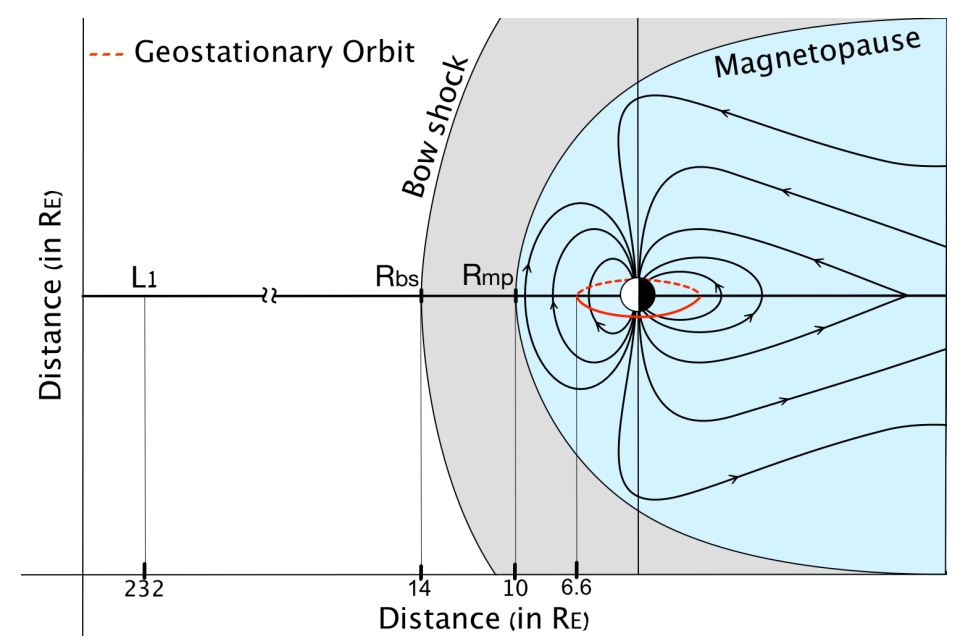

Figure 5. A schematic of the stand-off distance of the MP $\left(R_{\mathrm{mp}}\right)$ and BS $\left(R_{\mathrm{bs}}\right)$ in the GSM coordinate system. The dotted red circle in the equatorial plane represents the geostationary orbit at 6.6 earth radii. The L1 Lagrangian point of the sun-earth system is at 232 earth radii.

\subsubsection{Empirical model}

Lin et al. (2010), abbreviated as L10 hereafter, extended the assumptions of Shue et al. (1998) to address asymmetries and indentations near the polar cusps. Employing

a database of nearly 2708 MP crossings from observations by Cluster, Geotail, Goes, IMP8, Interball, LANL, Polar, TC1, THEMIS, WIND and Hawkeye, along with correspond- 
ing solar wind parameters from ACE, Wind and OMNI, they obtained a model for the MP which was parametrized by the solar wind dynamic and magnetic pressure $\left(\mathrm{P}_{d}+\right.$ $\left.\mathrm{P}_{m}\right)$, IMF- $\mathrm{B}_{z}$ and dipole tilt angle $(\psi)$, the dipole magnetic latitude of the subsolar point. Based on the relation between $\mathrm{P}_{d}$ and $\mathrm{r}_{m p}$, the influence of IMF $\mathrm{B}_{z}$ on $\mathrm{r}_{m p}$ (Shue et al., 1998) and the saturation effect of a southward IMF $\mathrm{B}_{z}$ on $\mathrm{r}_{m p}$ (Yang, Chao, Dmitriev, Lin, \& Ober, 2003), L10 expressed the stand-off distance for MP as:

$$
r_{m p}=a_{0}\left(P_{d}+P_{m}\right)^{a_{1}}\left(1+a_{2} \frac{\exp \left(a_{3} B_{z}\right)-1}{\exp \left(a_{4} B_{z}\right)+1}\right) R_{E}
$$

The coefficients $\left(a_{0}, a_{1}, a_{2}, a_{3}\right.$ and $\left.a_{4}\right)$ in equation (2) are obtained by using non-linear multi parameter fitting (Levenberg - Marquardt method) based on observations of 247 MP crossings that were found near the stand-off distance. The coefficients are listed in Table-2 of L10.

To obtain the MP shape, L10 expanded the eq. (1) as,

$$
r=r_{m p}\left\{\cos \frac{\theta}{2}+a_{5} \cdot \sin (2 \theta)[1-\exp (-\theta)]\right\}^{\beta} R_{E} .
$$

where the factor $1-\exp (-\theta)$ smooths out the MP shape near the subsolar point. The asymmetries and indentations are introduced through the azimuthal angle $\phi$, the angle between the projection of $\mathrm{r}$ in $\mathrm{Y}-\mathrm{Z}$ plane and the direction of positive $\mathrm{Y}$ axis. The flaring parameter $\beta$ (given by equation (5) of L10) is,

$$
\beta=\beta_{0}+\beta_{1} \cos (\phi)+\beta_{2} \sin (\phi)+\beta_{3} \sin ^{2}(\phi) .
$$

We considered the simpler case: $\phi=0$ (meridional plane) for which equation (4) reduces to $\beta=\beta_{0}+\beta_{1}$. $\beta_{0}$ and $\beta_{1}$ (eq. 16 and 17 of L10) are obtained using observations of $422 \mathrm{MP}$ crossings. The relevant values of the parameters are listed in Table -6 of L10.

The L10 model (eqs 2 and 3) yields good results in predicting the MP stand-off distance and shape. Also, when compared with the observed low latitude MP crossings, the standard deviation of the L10 model is the least $\left(0.54 R_{E}\right)$ amongst several other models (Jelínek et al., 2012). We, therefore, prefer Lin et al. (2010) model for the calculations of MP stand-off distance and shape.

\subsubsection{Numerical model}

To estimate the MP stand-off distance and shape using numerical calculations, we preferred the model by Lu et al. (2011), abbreviated as L11 hereafter, based on global MHD simulations that use the Space Weather Modelling Framework (SWMF), a framework for physics-based space weather simulations (Tóth et al., 2005). The functional form of Shue et al. (1998) was extended to describe the global MP size and shape using the method of multi-parameter fitting. L11 included azimuthal asymmetry via $\phi$ and extended the functional form shown in eq. (1). Thus, the dayside MP is given by,

$$
r=r_{m p}\left(\frac{2}{1+\cos \theta}\right)^{\alpha+\beta_{1} \cos \phi} R_{E} .
$$

where $\beta_{1}$ characterizes the azimuthal asymmetry with respect to $\phi$. Using the fitting results from Shue et al. (1997), the relationship between the $\left(\mathrm{r}_{m p}, \alpha, \beta_{1}\right)$ and solar wind conditions $\left(\mathrm{P}_{d}, \mathrm{~B}_{z}\right)$ were evaluated. The multiple parameter fitting results in the following best-fit functions (eq., 18, 19, 20 of L11):

$$
r_{m p}= \begin{cases}\left(11.494+0.0371 B_{z}\right) P_{d}^{-1 / 5.2}, & B_{z} \geq 0 \\ \left(11.494+0.0983 B_{z}\right) P_{d}^{-1 / 5.2}, & B_{z}<0\end{cases}
$$




$$
\begin{gathered}
\alpha=\left\{\begin{array}{l}
\left(0.543-0.0225 B_{z}+0.00528 P_{d}+0.00261 B_{z} P_{d}\right), \text { for } B_{z} \geq 0 \\
\left(0.543+0.0079 B_{z}+0.00528 P_{d}-0.00019 B_{z} P_{d}\right), \text { for } B_{z}<0
\end{array}\right. \\
\beta_{1}=\left\{\begin{array}{l}
\left(-0.263+0.0045 B_{z}-0.00924 P_{d}-0.00059 B_{z} P_{d}\right), \text { for } B_{z} \geq 0 \\
\left(-0.263-0.0259 B_{z}-0.00924 P_{d}+0.00256 B_{z} P_{d}\right), \text { for } B_{z}<0
\end{array}\right.
\end{gathered}
$$

The model results by L11 yield good matching when compared with the high and low latitude MP crossings. So we prefer to use the numerical model by L11.

\subsection{BS stand-off distance}

We briefly describe below the models used in the calculations of the BS stand-off distance. The first model, Jelínek et al. (2012) represents an empirical approach while the second one, J. F. Chapman and Cairns (2003), represents a numerical approach.

\subsubsection{Empirical model}

The model by Jelínek et al. (2012), hereafter abbreviated as J12, investigated the solar wind, magnetosheath and magnetosphere using measurements from the THEMIS and $A C E$ spacecrafts. J12 assumed a parabolic shape for the BS, which is rotationally symmetric around the $X_{G S M}$ coordinate system. The authors used an analytic expression, given by

$$
r_{b s}=15.02 P_{d}^{-1 / 6.55} R_{E} .
$$

to estimate the BS stand-off distance, where the free parameters are generally determined using a least square fitting to the full data set. The equation (9) does not take into account the Mach number, however, these simple model results are found to be in good agreement when compared with more than 6000 spacecraft crossings of the BS region. We therefore used the model by Jelínek et al. (2012) to compute the BS stand-off distance.

\subsubsection{Numerical model}

The model by J. F. Chapman and Cairns (2003), hereafter abbreviated as CC03, uses three dimensional MHD simulations from Cairns and Lyon (1995). The model uses parameters such as $\mathrm{P}_{d}$, the Alfvén Mach number $\left(\mathrm{M}_{A}\right)$ and the orientation of the IMF $\left(\theta_{I M F}\right)$ with respect to the solar wind velocity $\left(v_{s w}\right)$ direction. J. F. Chapman and Cairns (2003) mainly considered two special cases of $\theta_{I M F}=45^{\circ}$ and $90^{\circ}$, of which we use $\theta_{I M F}=$ $90^{\circ}$ to estimate the BS stand-off distance given by

$$
r_{b s}=\left(\alpha_{0}+\frac{\alpha_{1}}{M_{A}}\right)\left(\frac{P_{d}}{1.87}\right)^{-1 / 6} R_{E} .
$$

Here $\left(\alpha_{0}, \alpha_{1}\right)$ are the fitting parameters obtained by a least square fitting to the simulated BS locations. The CC03 model results have been compared with the available spacecraft crossings close to the nose region of the BS and it is found that for the near-Earth regime $\left(-20 R_{E}<x<35 R_{E}\right)$, the model predicted the BS location very well.

\section{Data and analysis}

For the present study, in order to compute the stand-off distances of the MP and $\mathrm{BS}$, we use daily averaged measurements of solar wind dynamic pressure and IMF into equations $2,6,9$, and 10 described in $\S 3.1$ and $\S 3.2$. The solar wind dynamic pressure was derived using solar wind proton density $\left(N_{p}\right)$ and solar wind velocity $\left(v_{\mathrm{sw}}\right)$. The data for $N_{p}, v_{\mathrm{sw}}$, and IMF for the period Feb. 1975-Dec. 2017 were obtained from the OMNI data base (https://omniweb.gsfc.nasa.gov/). OMNI data are available as low resolution and high resolution OMNI data sets. For the high resolution data (1-min and 
5-min averages), interpolations are usually performed on the phase front normal directions for gap intervals of less than 3 hours, and for the time shift for gap intervals of less than one hour. So for this study, we used the low resolution (available as hourly, daily and 27-day averages) data for which no interpolation was performed. Actually, the low resolution OMNI data base is a compilation of hourly averaged near-earth solar wind magnetic field data and various other solar wind plasma parameters from several spacecrafts at geocentric or L1 orbit which have been extensively cross calibrated, and, for some spacecraft and parameters, cross-normalized (https://omniweb.gsfc.nasa.gov/html/ow_data .html). The available daily average OMNI data are simple average of these hourly averaged values. We directly used in our calculations the available daily averaged measurements of IMF and solar wind plasma parameters from OMNI data base by excluding the days with missing values and days that have less than 8 hourly average values of IMF and solar wind plasma parameters. In the next step, from the computed daily averaged values of MP stand-off distances, we obtained the Carrington rotation (27.2753 days) and annual averaged values of MP stand-off distances. In addition, we also used the monthly averages of IMF- $B_{z}, \mathrm{P}_{d}$ and MP stand-off distances to make a time series forecast using a Auto Regressive Integrated Moving Average (ARIMA) method (see section $§ 5.2$ and Appendix A).

The stand-off distances of the MP and $\mathrm{BS}$ are sensitive to the variations in $\mathrm{P}_{d}$ and $\mathrm{IMF}-\mathrm{B}_{z}$. They are also affected by the Alfvén and magnetosonic Mach numbers. The models that we used in this study mostly consider these parameters while estimating the stand-off distances of MP and BS. The angle between the magnetic field and the solar wind velocity vector is critical in determining the shock location of the BS (Cairns \& Lyon, 1995). As mentioned earlier, the shape of the MP is asymmetric due to the cusp regions. We, therefore, used models with an analytical formula, taking into account the pressure imbalance due to the Earth's dipole tilt angle $(\psi)$ that reproduce the cusp regions resulting in an asymmetric MP shape (Lin et al., 2010; Shukhtina \& Gordeev, 2015). Zhong et al. (2014) have shown that the earth's dipole moment has been decaying over the past 1.5 centuries. Even assuming a linear rate of decay to persist their results suggest that the average stand-off distance of the MP would only move $\sim 0.3 R_{E}$ towards the earth per century. Since in the present study, we are looking for the long term trend in the change of MP and BS stand-off distances, so the contribution to the MP position and shape at the subsolar point due to the change in the pressure caused by the declining dipole moment will be small. Thus, we neglected the effect of the dipole tilt angle and for the present study, we consider the MP and BS to be symmetric about the sun-earth line, i.e. around the X-axis of the GSM coordinate system. Further, for calculating the BS stand-off distance using the numerical model by CC03 we kept the IMF angle $(\theta)$ fixed at $90^{\circ}$.

\section{Results}

It is to remind here that we discuss in the following subsections about the temporal variations of daily averaged values of MP and BS stand-off distances which directly obtained using daily averaged IMF and solar wind plasma parameters in numerical and empirical models as described in section 3.1 and section 3.2. We further averaged the values of MP stand-off distances covering one Carrington-rotation (27.2753 days) and also obtained the annual averages to show the temporal variations in MP stand-off distance and MP shape.

\subsection{Position of MP and BS}

Figure 6 shows the computed stand-off distance of the MP during the period Jan. 1975-Dec. 2017. The top panel shows the result obtained by using the empirical model 

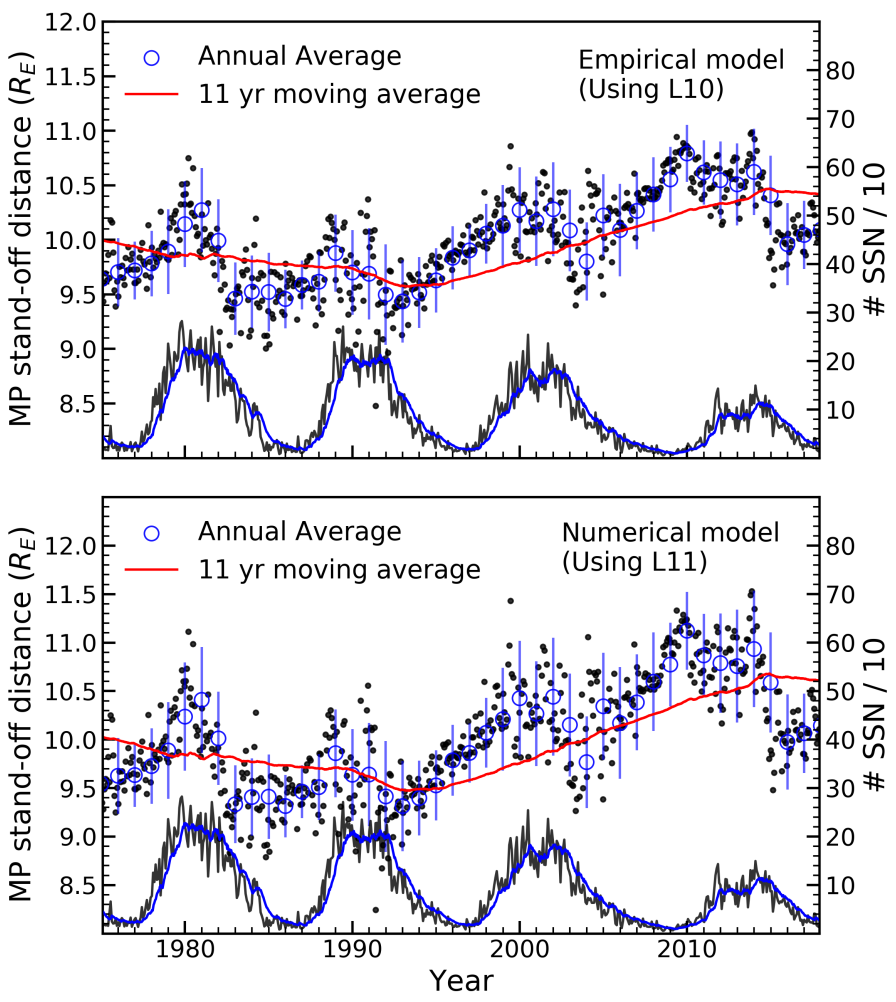

Figure 6. Carrington-rotation averages of MP stand-off distance for the period Feb. 1975-Dec. 2017 (grey filled circles) derived using Lin et al. (2010) (L10) (upper panel) and Lu et al. (2011) (L11) (lower panel). The blue circles represent annual averages shown with $1 \sigma$ error bars. The red line is a eleven year moving average of the daily average of MP stand-off distance. The monthly averaged sunspot numbers, scaled down by a factor of 10 , is shown by the solid curve in grey with the smoothed values (one year moving average) overplotted in blue.

L10 while the results in the lower panel are derived using the numerical model L11. The black filled dots are the Carrington-rotation averages of MP stand-off distances, while the open blue circles represent the annual means of MP stand-off distance shown with $1 \sigma$ error bars. Shown at the bottom of each panel, by a grey solid line overplotted in blue with the smoothed value (of one year moving averages), is the monthly averaged smoothed sunspot number, scaled down by a factor of 10. It is clearly seen from both panels of Fig.6 that the MP stand-off distance is modulated by the solar cycle activity, having roughly a periodicity of 11 years. The comparison of MP stand-off distance of each solar cycle reveals that the MP stand-off distance starts to increase when the sunspot cycle is roughly in the declining phase and it continues to increase until the approach of the sunspot cycle maximum. This is much clearer during the minima of solar cycles 22 and 23 when we see a clear rise in stand-off distance. Besides this solar cycle modulation, we can see a long term increasing trend in the MP stand-off distances since the declining phase of solar cycle 22, around the mid-1990's, in sync with the declining trend of the diminishing sunspot cycles 22-24. To remove the 11-year solar cycle modulation and investigate the increasing trend of MP stand-off distance, the daily average of MP stand-off distance was smoothed using a eleven year running mean, shown by the overplotted solid red curve in both panels of Fig. 6. A clear increasing trend in the MP stand-off distance is evident from the overplotted red curve which begins to rise around the mid-1990's. This increase was found to be $\sim 15 \%$, irrespective of the empirical or numerical model used. 
Figure 7 shows the BS stand-off distances obtained by using the empirical model (J12, $\S 3.2 .1)$ (left panels) and the numerical model (CC03, §3.2.2) (right panels). The upper panel of Fig.7 shows the daily averaged value of the normalized BS stand-off distances from Feb. 1975 to Dec. 2017. For Figure 7, we computed the daily BS stand-off distance after normalizing by the average value of BS stand-off distance at typical solar wind conditions at $1 \mathrm{AU}\left(\mathrm{P}_{d} \sim 1.87 \mathrm{nPa}, \mathrm{B}=7 \mathrm{nT}, \mathrm{N}_{p}=6.6 \mathrm{~cm}^{-3}, \mathrm{~V} \sim 440 \mathrm{~km} / \mathrm{s}, \mathrm{M}_{a}=8\right)$. Thus, the normalized BS stand-off distance can be less than unity. However, in Figure 7 (upper panels), we presented only the normalized BS stand off distance larger than unity. In this way, it is convenient to identify the effects of the long term solar activity on the BS stand-off distance, which is the main goal in this study. The difference in the magnitude of the BS stand-off distance estimated by the two models can be understood in the following way. Both the models, CC03 and J12 relate the BS stand-off distance with solar wind dynamic pressure as a power law, but with different power law indices. Also, the CC03 model includes the effect of the Alfvén Mach number $\left(\mathrm{M}_{A}\right)$, which has been neglected in J12. It is clear that irrespective of the models used, the normalized BS stand-
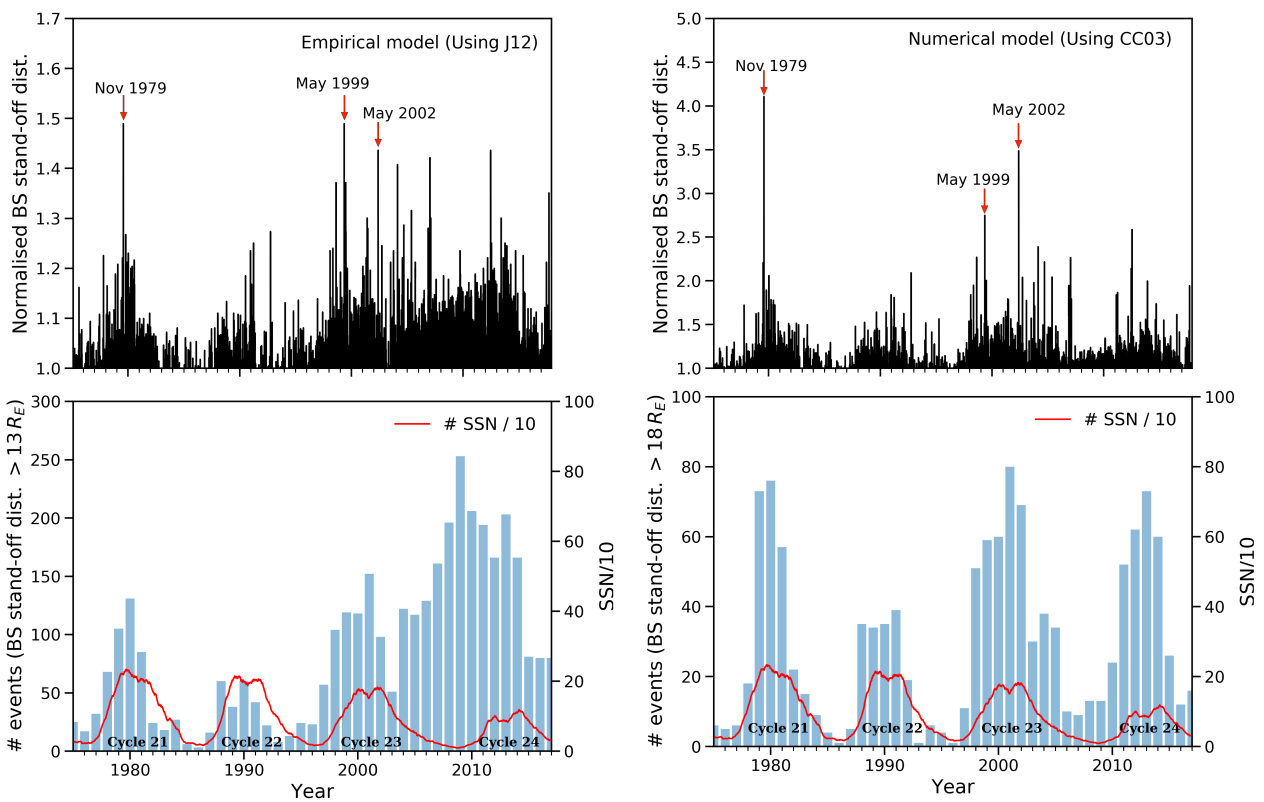

Figure 7. (Top) Daily averages of normalized BS stand-off distance between Feb. 1975 and Dec. 2017. Three extreme events, designated as solar wind disappearance events in the text, have been labelled with the event dates. (Bottom) The distribution of the number of events or instances for which the BS stand-off distance > average stand-off distance between 1975 and 2017. A 12 month moving average of sunspot numbers, scaled down by a factor of 10 is overplotted in red. Left panels uses J12 model while right panels uses CC03 model.

off distances show a large number of cases of the BS stand-off distance extending well beyond the average value. Three such events are indicated in Fig.7 (upper panel). These events have been well studied and are referred to as solar wind disappearance events (Balasubramanian, Janardhan, Srinivasan, \& Ananthakrishnan, 2003; Janardhan, Fujiki, Kojima, Tokumaru, \& Hakamada, 2005; Janardhan, Fujiki, et al., 2008; Janardhan, Tripathi, \& Mason, 2008) due to the extremely low densities observed at $1 \mathrm{AU}\left(<0.1 \mathrm{~cm}^{-3}\right)$ for periods exceeding 24 hours. During all three solar wind disappearance events, a sharp decrease in $P_{d}(<0.02 \mathrm{nPa})$ was seen indicating the sensitive response of the BS standoff distances to the changed solar wind conditions. Also, it is seen from Fig.7 (upper panel) 
that the normalised BS stand-off distance, on an average, follows the 11-year solar cycle. However, a careful inspection of the model results shows that the solar cycle modulation is very clear when we used the model by CC03. While the results obtained by using J12 show large excursions of the normalised BS stand-off distance extending well below the average value during cycle 23 minimum. It is to be noted that solar cycle 23 witnessed an unusual and deep solar minimum during the same period. It appears that there is a difference in the model results for the normalized BS stand-off distance during cycle 23 with the empirical model calculations showing more number of days having an increased BS stand-off distance than the numerical model calculations.

To further quantify the effect of solar wind conditions on the BS, we selected those days with normalized values of BS stand-off distance which are more than $1 \sigma$ above the average BS stand-off distance at typical solar wind conditions, which we referred to as an event. For typical solar wind conditions at 1 AU, the average BS stand-off distance according to J12 is $13 R_{E}$ and that according to CC03 is $18 R_{E}$. The lower panels of Fig.7 shows the histogram of the distribution of the number of events for the period 1975-2017 obtained using the empirical model by J12 (lower left) and the numerical model by CC03 (lower right). As stated earlier, it is clear that the BS excursions beyond the average BS stand-off distance are observed consistently for each solar cycle and we see a clear modulation of solar cycle for solar cycles 21-23, irrespective of the model used. In order to show the solar cycle modulation, we overplotted, in red, the 12-month moving average of the smoothed sunspot number, scaled down by a factor of 10, for the period 1975-2017, as shown at the bottom of each of the lower panels of Fig.7. As pointed out earlier, we see a difference in the number of events during the solar cycle 23 minimum for the results obtained by the empirical model J12. Also, evident from the lower panels of Fig.7 is that there is a significant increase in the number of events with the BS stand-off distance exceeding well below the average value since around mid-1990's. One needs to bear in mind, that at the same time we have seen solar photospheric magnetic fields showing a steady decline. In case of both the models, the increase, that we see in the number of events after mid-1990's is found to be more than $\sim 40 \%$ when compared with the number of events before mid-1990's.

\subsection{Shape of MP}

Using the standard GSM coordinate system we computed the position of MP, $X_{s}=$ $r \cos (\theta)$ (the stand-off distance along the Earth-Sun line for the day-side MP) and $R_{s}=$ $\left.r \sin (\theta)=\sqrt{(} Y^{2}+Z^{2}\right)$ (in GSM coordinate, also known as the transverse radius), where $\theta$ is the solar zenith angle. In order to compute the shape of MP at solar minima for cycles 20-23, we plotted $R_{s} \mathrm{v} / \mathrm{s} X_{s}$, averaged over 1 year intervals for years 1976, 1986, 1996 and 2008 during each solar minimum for cycles 20, 21, 22 and 23, respectively. The 1year interval corresponds to the period shown by grey vertical bands in Fig.2. We refer to the plot of $R_{s} \mathrm{v} / \mathrm{s} X_{s}$ as the MP shape. Figure 8 shows the plot of $R_{s} \mathrm{v} / \mathrm{s} X_{s}$ for a solar zenith angle between $\theta=0^{\circ}$ and $\theta=90^{\circ}$. The upper panel of Fig. 8 shows the MP shape obtained using the eq. (3) (§3.1.1) based on the empirical model by L10, while the lower panel shows the MP shape obtained using the eq. (5) (§3.1.2) based on the numerical model by L11. The 1-year averaged MP shape is labelled by the year, for e.g. the MP shape of 1976, shown by the solid black curve, refers to the MP shape that is averaged over 1 year in 1976 during the solar minimum of cycle 20. Similarly, the solid curves in indigo, red and green represent 1 year averaged MP shape for years 1986, 1996 and 2008, for solar minima of cycles 21, 22 and 23, respectively. Also, an inset plot presenting the zoomed in MP shape is shown in each panel of Fig.8 that shows clearly the expansion of MP shape. It is evident from Fig.8 that in both cases (for L10 (upper panel) and L11 (lower panel) models) the average MP shape in 1986 for the solar minimum of cycle 21 falls below the average MP shape in 1976 for the solar minimum of cycle 20, and then bounces forward in 1996 and continues to expand until 2008. So the 1 year averaged MP shape during solar minima of cycles 22-23 showed a continuous expansion. 
For the period between 1996 and 2008, the stand-off point expanded by nearly 1 $\mathrm{R}_{E}$ from $\sim 9.8 \mathrm{R}_{E}$ to $\sim 11 \mathrm{R}_{E}$, irrespective of the model used. We have also computed the MP flaring angle that is a function of $P_{d}$ and IMF- $B_{z}(\mathrm{Lu}$ et al., 2011). We have found that the flaring angle also shows a 11 year solar cycle modulation (Figure not shown here) and when this modulation is removed by smoothing the flaring angle with a 11 year moving average, we see a clear increasing trend since 1996.



Figure 8. The one year averaged MP shape at solar minima for cycles 20-24 shown by a plot of transverse radial distance of MP, $R_{s}$ against the extent of the MP along the sun-earth line, $X_{s}$. The upper panel uses Lin et al. (2010) (L10) (§3.1.1) and the lower panel uses Lu et al. (2011) (L11) (§3.1.2). The solid black line is the one year interval averaged of MP shape for the year 1976 during the minimum of cycle 20. Similarly, the pink, red and green solid curves represent 1 year averaged MP shapes for years 1986, 1996 and 2008 during solar minima of cycles 21, 22 and 23, respectively. The blue curve presents the forecast of MP shape in the year 2020, with a shaded blue band of $95 \%$ confidence interval, for the minimum of the current cycle 24 . Also, an inset plot presenting the zoomed in MP shape is shown in both upper and lower panels.

Under the possibility of having a continuing declining trend in solar polar fields at least until 2020, it is imperative to know how the Earth's magnetosphere will look like in 2020. So we forecast the shape of the MP for the year 2020 during the forthcoming minimum of cycle 24. As stated earlier, the MP shape is sensitive to the changes in $\mathrm{P}_{d}$ and $B_{z}$ (see equations 3 and 5 ). We, thus, carried out a systematic exercise to identify the trend and seasonality in the time series data of $\mathrm{P}_{d}$ and $B_{z}$ using a Auto Regressive Integrated Moving Average (ARIMA) process (see Appendix A). This enables us to ob- 
tain a stationary time series facilitating further analysis and to make a forecast. The forecast is obtained for monthly averages of $B_{z}, P_{d}$ and $r_{m p}$ for the time period between Dec. 2017 and Dec. 2020 (see Figures in Appendix A). We compared the predicted values of $P_{d}$ and $r_{m p}$ by the ARIMA method with the observed values of $P_{d}$ and the estimated values of $r_{m p}$ obtained from the OMNI2 data base between the period 2018-01-01 and 2019-03-01. It is evident from the forecast of $P_{d}$ and $r_{m p}$ that the predicted and the observed values between the period 2018-01-01 and 2019-03-01 are in good agreement. This verifies the ARIMA method. It is, however, not the case for $B_{z}$ which shows a simple linear extrapolation curve without any variations. However, using the ARIMA method the value of $B$ in 2020 has been estimated to be $\sim 5.4( \pm 0.9) \mathrm{nT}$. Using the correlation between $\mathrm{B}$ and solar polar fields, we also estimated a value of $5.3( \pm 0.3) \mathrm{nT}$ at 2020 for the value of $B$. The values of $\mathrm{B}$ computed from these two separate methods agree well within uncertainty. This further validates the forecast using the ARIMA method. The curve in blue in Fig. 8 depicts the forecast of the MP shape for 2020 for the minimum of cycle 24. The blue band in Fig. 8 signifies the region of $95 \%$ confidence interval around the MP shape of 2020. It is clearly seen from Fig.8 (both upper and lower panels) that the MP shape in 2020 shows further expansion than in 1996 (during solar minima of cycle 22). However, it resumes an average value of $10.3 R_{E}$, which is less than that in 2008 (during solar minima of cycle 23).

\section{Discussion}

In the present study, we quantified the response of the earth's magnetosphere via the variations in the stand-off distance of the MP and BS for the period February 1975December 2017 caused by the long term changes that have been seen in solar photospheric magnetic fields, inner heliospheric solar wind micro-turbulence levels and solar wind changes at 1 AU, since the mid-1990's. We carried out the computation of MP and BS standoff distances as well as the MP shape using both empirical and numerical models and compared the model results. The MP stand-off distance and the MP shape was computed using L10 (empirical model, §3.1.1) and L11 (global MHD simulations, §3.1.2). For the BS stand-off distance we used the model by CC03 (based on global MHD simulations, $\S 3.2 .2$ ) and compared them with the results obtained by J12 (empirical model, §3.2.1).

Our analysis showed a continuation of the steady decline until 2017 in the highlatitude photospheric magnetic fields and solar wind micro-turbulence levels in the inner heliosphere. Also, a similar behaviour of global reduction in values of solar wind parameters at $1 \mathrm{AU}$ such as $\mathrm{B}, \mathrm{N}_{p}, \mathrm{v}, \mathrm{P}_{d}$ and $\mathrm{He}^{++} / \mathrm{H}^{+}$post mid-1990's was clearly seen. Corresponding to this observed decline, a steady increase of $\sim 15 \%$ was observed in the stand-off distance of the MP, irrespective of the model used. Our result of the increase in the MP stand-off distance is consistent with the increase in the stand-off distance of the MP reported by McComas et al. (2013) wherein, the authors found an increase in the MP stand-off distance from $10 R_{E}$ in 1974-1994 to $11 R_{E}$ in 2009-2013. From our study, the value of the stand-off distance of MP from 1974-1994 were found to be 9.7 $\mathrm{R}_{E}$. In contrast, the value of MP stand-off distance from 1995-2017 were found to be $10.7 \mathrm{R}_{E}$. So McComas et al. (2013) and our study show a similar increase of about $1 R_{E}$ in MP stand-off distance. We also observed a significant increase of more than $40 \%$ in the number of events (after 1995) where the stand-off distance of the BS exceeded the average stand-off distance over the past $\sim 24$ years, when compared with the number of events prior to 1995 .

It is to be noted that both $\mathrm{P}_{d}$ and IMF magnitude have shown a similar temporal behaviour (Jian, Russell, \& Luhmann, 2011; McComas et al., 2013, 2003; Richardson, Wang, \& Paularena, 2001) with a global reduction of $\sim 40 \%$ in their average values over the past 24 years. In our analysis, we showed this behaviour of $\mathrm{P}_{d}$ and IMF that is continuing till date. Generally, during solar minimum, photospheric high latitude magnetic fields extend to low latitudes and are then pulled into the heliosphere by the so- 
lar wind thereby, forming the IMF (Schatten \& Pesnell, 1993). The changes in the solar wind conditions such as decline in $\mathrm{P}_{d}$ and IMF strength can thus be interpreted as being induced by global changes in solar photospheric magnetic fields. We also showed this causal link of $\mathrm{P}_{d}$ and IMF with solar polar fields in our analysis. The computed correlations of $\mathrm{P}_{d}$ and IMF with solar polar fields, $\mathrm{B}_{p}$ over the past four solar cycles apparently proved this connection between them, which has been found to be better if the correlation is considered for only the minima of cycles 23 and 24, i.e., post mid-1990's (with correlation coefficients of 0.95 and 0.92 for $\mathrm{P}_{d}$ and IMF with $\mathrm{B}_{p}$, respectively). A decrease in $\mathrm{P}_{d}$ and IMF causes an expansion of the BS and MP, resulting in an increase in their sub-solar distances. The stand-off distance of the MP, in general, exhibits a power law dependence on the dynamic pressure, with power law index $\sim-1 / 6$ (Mead \& Beard, 1964; Petrinec \& Russell, 1996). A self-similar scaling suggests an identical power law dependence for the stand-off distance of the BS (Cairns \& Lyon, 1996). As mentioned earlier, the models used for the computation of the stand-off distances of BS and MP are parametrized by the changes in both $\mathrm{P}_{d}$ and the IMF, so a global rise in the standoff distance of MP and BS is thus expected considering the global reduction in both $\mathrm{P}_{d}$ and the IMF. We checked the values of $\mathrm{P}_{d}$ from 1974-1994 and 1995-2017, which were found to be, respectively, $\sim 2.9 \mathrm{nPa}$ and $\sim 2.0 \mathrm{nPa}$, showing a decrease of $\sim 31 \%$ post1995 in comparison to pre-1995. The corresponding increase of $\sim 9.3 \%$ observed in the stand-off distance of the MP can thus be attributed to the power law dependence of the MP stand-off distance on the $\mathrm{P}_{d}$. However, the power law index found in several empirical as well as numerical studies is little less than $-1 / 6$ (Lin et al., 2010; Shue et al., 1998), which is the theoretically predicted value for a dipole in vacuum. This deviation from $-1 / 6$ can probably accounted by a plasma pressure inside the magnetosphere (Jelínek et al., 2012). The increasing trend in stand-off distances of the MP and BS is therefore consistent with the ongoing declining trend in high- latitude photospheric magnetic fields and solar wind micro-turbulence levels that we have seen during this last 22 years. This indicates that the earth's magnetosphere is very sensitive to the changes in solar wind conditions modulated by changes in solar magnetic activity.

From our study, we found that the strength of IMF and $\mathrm{P}_{d}$ averaged over 1 year intervals during solar cycle minima showed a better correlation with solar polar fields. Based on this correlation, we also studied the variation in the average MP shape for several 1-year intervals during solar minima of cycles 20-23 and found that the average MP shape in 1996 during the minimum of cycle 22 has shown an outward expansion, which has undergone a further significant expansion in 2008 during the minima of cycle 23, which happened to be one of the deepest experienced in the past 100 years. Our results showed an average increase in the MP shape of $>1 R_{E}$ for the period 1996-2008. Based on the assumption that the steady decline in solar high-latitude fields would continue, we predicted the shape of MP for the expected minimum of cycle 24 in 2020 using the forecast values of $B_{z}, P_{d}$ and $r_{m p}$ for the time period between Dec. 2017 and Dec. 2020 obtained by the ARIMA method. The predicted shape of the MP in 2020, during the expected minimum of solar cycle 24, suggests an expanded terrestrial magnetosphere with the MP shape in 2020 being larger than the MP shape during the minimum of cycle 22 and smaller than the MP shape during the minimum of cycle 23.

The comparison of the model results for the long term trend in the MP stand-off distance and shape, in general, show they do not depend on the model used. While the comparison of the model results for the long term trend in the BS stand-off distance also show that they are independent of the model used for solar cycles 21-22. However, they show a difference for the BS stand-off distance for the period of solar cycles 23-24.

The location of the MP inside the position of geostationary orbit is crucial since the MP acts as a shield against high speed solar wind during geomagnetic storms caused by CME impacts on the magnetosphere. These impacts, if they originate from a large high velocity CME have the ability of compressing the MP to distances below the geo- 
Table 1. Daily averaged values of MP Stand-off distance $\left(r_{m p}\right.$ in $\left.R_{E}\right)$ closest to geostationary orbit (6.6 $\mathrm{R}_{E}$ ) using models due to Lin et al. (2010) (L10) and Lu et al. (2011) (L11)

\begin{tabular}{lccc}
\hline Date & $\begin{array}{c}r_{m p} \text { in } R_{E} \\
\text { Using L10 }\end{array}$ & $\begin{array}{c}r_{m p} \text { in } R_{E} \\
\text { Using L11 }\end{array}$ & $\begin{array}{c}\text { Duration } \\
(\sim \text { hours })\end{array}$ \\
\hline $1978-06-02$ & $6.62 \pm 0.16$ & $6.31 \pm 0.18$ & 11 \\
$1991-06-05$ & $6.83 \pm 0.20$ & $6.51 \pm 0.22$ & 10 \\
$2005-01-17$ & $6.99 \pm 0.15$ & $6.84 \pm 0.16$ & 09 \\
\hline
\end{tabular}

stationary orbit, which lies at a distance of around $6.6 R_{E}$, thereby exposing the geo-stationary orbit to high energy particle flux from the sun and posing a threat of causing damage to or destroying satellite systems entirely. We list in Table 1 the events when the daily values of MP stand-off distance comes close to the geostationary orbit $\left(\sim 6.6 R_{E}\right)$, computed using the L10 and L11 models separately. The solar wind dynamic pressure corresponding to the $\mathrm{MP}$ stand off distance $=6.6 \mathrm{R}_{E}$ is approximately $23 \mathrm{nPa}$. Form Table 1 , it is seen that there were two events between the year 1968 and 1995 in contrast to the single event post 1995. The data also showed four other events between 1968 and 1995, but since there were large data gaps in these data sets, they have not been included in Table 1. The less number of events with MP stand-off distance less than $7.0 \mathrm{R}_{E}$ post mid-1990's agrees with the observed increasing trend of MP stand-off distances post mid1990's.

Our study shows the steady decline in high-latitude photospheric fields and solar wind micro-turbulence levels have been continuing until December 2017, the end of our data set. On the assumption that the decline would continue until 2020, the expected minimum of the current solar cycle 24. Janardhan et al. (2015) predicted that the next solar cycle 25 would be a weaker cycle with a maximum smoothed sunspot number (SSN) of $62 \pm 12$ on the old unrevised scale of sunspot number (V1.0). Other researchers have also made similar predictions of a weaker solar cycle 25 (Bhowmik \& Nandy, 2018; Cameron, Jiang, \& Schüssler, 2016; Upton \& Hathaway, 2018). Based on the hemispheric asymmetry of solar polar field reversal in cycle 24 and the prolonged, 2.5 year long, zero-field condition before the completion of polar field reversal in the solar northern hemisphere, Janardhan et al. (2018) predicted a weaker cycle 25, similar to cycle 24. In a paper (Bisoi et al., submitted manuscript, 2019), also predicted a weaker cycle 25 with a maximum SSN of $132 \pm 11$ on the revised scale of sunspot number (V2.0), which is slightly stronger than cycle 24. Based on the continuous declining trends of solar high-latitude fields for the past 25 years and the prediction of a weaker cycle 25, we expect a low level of sunspot activity in future beyond solar cycle 25, if the declining trends continue beyond 2020, a condition akin to the Maunder minimum (1645-1715) when the sunspot activity was extremely low or almost non-existent. Other researchers have also arrived at similar conclusion and reported that the Sun may move into a period of very low sunspot activity comparable with the Dalton minimum Zolotova and Ponyavin (2014), while (SánchezSesma, 2016; Zachilas \& Gkana, 2015) claim that the Sun is moving into another extended Maunder-like minimum. However, we have not carried out any extensive analysis to show the long term forecasts of Maunder minimum like conditions. Our interpretation is purely based on the assumption of a continuation of declining trend of solar high-latitude photospheric fields. The current understanding is that sunspot fields are generated by a shearing of pre-existing poloidal fields (high latitude fields) and the regeneration of poloidal fields through a Babcock-Leighton mechanism with the whole process operated by an interior solar dynamo. The strength of poloidal fields depends on the large scale scatter in the sunspot fields and the tilt angle distribution of bipolar sunspot regions (D'Silva 
\& Choudhuri, 1993). This is a random process. We have seen continuing weaker polar fields in cycles 22 and 23. The same is also seen in cycle 24 that is continuing till date. The probability for a random process of polar field regeneration yielding three successive weaker cycles is clearly very low and therefore not only extremely unusual, but points to a lack of a complete understanding of the solar dynamo process. Thus, we expect a continuation of the decline in solar high-latitude fields in future solar cycles, that could result in very low sunspot activity in future and possibly result in conditions akin to the Maunder minimum. However, the long term decline in solar fields could represent a period of solar activity with a period longer than 11 years. As of now, we have solar magnetic field measurements only for the last four solar cycles, cycles 21-24, it is difficult to determine the longer periods than the known period of 22 years (or a solar magnetic cycle) in solar high-latitude magnetic fields. Also, the solar high-latitude fields have been showing a long term decline more than 25 years (greater than the solar magnetic cycle of 22 years). We, therefore, expect a continuation of the decline of high-latitude fields beyond solar cycle 24 .

Using a global thermodynamic model, Riley et al. (2015) reported the likely state of the solar corona, during the later period of the Maunder minimum, devoid of any large scale structure and driven by a reduced photospheric magnetic field strength. The photospheric field strength during the last two solar cycles has been steadily decreasing (since $\sim 1995$ ) and the trends indicate that it is likely to decline in the same manner in future solar cycles. This implies a state of corona with no large scale structure much alike Maunder minimum period, which in turn, leads to an expansion of terrestrial magnetosphere with an increased stand-off distance for the BS and the MP.

\section{Conclusions}

Our study underlines the causal relationship between solar activity changes and the corresponding global response of the earth's magnetosphere via the variations quantified by the stand-off distances of the BS and MP. Our study mainly showed an increasing trend in the stand-off distance of MP and BS corresponding to the observed decreasing trend in solar high-latitude photospheric polar fields and solar wind micro-turbulence levels since the mid-1990's. A similar increasing trend was also observed for the MP shape till 2008 which is the minimum of the solar cycle 23. However, a forecast of the MP shape in 2020, the expected minimum of cycle 24, showed a smaller MP stand-off distance. Further, we find two instances between 1968 and 1991 when the MP stand-off distance reach closest to 6.6 Earth radii (the geostationary orbit) for duration ranging from $9-11$ hours and a single event in 2005, after the start of the decline in photospheric fields began. Finally, the decline in photospheric fields has now continued for over two solar cycles or one full magnetic cycle of 22 years. If the declining trend continues beyond 2020, a Maunder minimum like condition may be expected beyond solar cycle 25 .

Continued investigation to understand and forecast the influence of solar activity on the near earth environment and the ecosystem is, therefore, of considerable importance.

\section{A : Forecasting a time series}

Auto-Regressive Integrated Moving Average, $\operatorname{ARIMA}(p, d, q)$, is a general class of models for forecasting a stationary time series. A non-seasonal ARIMA forecasting equation for a time series is a linear equation given by (Brownlee, 2017),

$$
Y_{t}=\mu+Y_{t-d}+\sum_{i=1}^{p} \phi_{i} Y_{t-i}-\sum_{j=1}^{q} \theta_{j} \epsilon_{t-j}
$$


Where, $\mu$ is a constant, second term represents $d$ number of non-seasonal differences needed to make time series stationary. Third term is a weighted $\left(\phi_{i}\right)$ auto-regressive process of the order of $p$ and fourth term corresponds to a weighted $\left(\theta_{i}\right)$ average of the noise (moving average process) of the order of $q$.

To forecast the shape of the MP based on the models due to L10 and L11, we need to forecast two parameters, namely - solar wind magnetic field $\left(B_{z}\right)$ and dynamic pressure $\left(P_{d}\right)$. Using the forecast for $B_{z}$ and $P_{d}$, we can derive the forecast of the MP standoff distance $\left(r_{m p}\right)$. This then yields the shape of the MP Following steps are involved in obtaining a forecast (Brownlee, 2017):

1. Decomposition of time series

2. Analysis of stationarity

(a) Moving average and standard deviation

(b) Augmented Dicky-Fullar test

3. Making a time series stationary

4. Determine the parameters of ARIMA

(a) Auto-correlation function (ACF) and Partial Auto-correlation function (PACF)

(b) Akaike information criterion (AIC)

5. Time series forecast using the best fit parameters determined in step 4 .

The decomposition of the time series $\left(B_{z}\right.$ and $\left.P_{d}\right)$ into trend, seasonal variations and residue has been carried out. We found that $P_{d}$ and $B_{z}$ shows modulation in response to 11 year sunspot cycle. 11 year moving average reveals an increasing trend in $P_{d}$, with seasonal variations of the period of one year. $B_{z}$, on the other hand, shows dominant stochastic behaviour throughout. We applied Seasonal ARIMA (SARIMA) method to $B_{z}$ and $P_{d}$ in order to obtain the forecast. This requires to estimate the hyper-parameters $\mathrm{P}, \mathrm{D}$ and Q corresponding to the seasonal components of the parameters of ARIMA (p, d, q). This is generally represented as $(p, d, q) \times(P, D, Q, m)$ where $\mathrm{m}$ is the period of seasonal variations.

The analysis of stationarity shows that all three parameters $\left(B_{z}, P_{d}\right.$ and $\left.r_{m p}\right)$ follow 11 year sunspot cycle. Also 11 year moving average shows decreasing trend in $B_{z}$ and $P_{d}$ and increasing trend in $r_{m p}$ since $\sim 1995$. We considered Augmented Dicky-Fullar test $(\mathrm{ADF})$ to determine the stationarity of the time series. In all three cases we find that after applying first order difference we get test statistics (ADF-test) well below the $5 \%$ critical value with no trend in 11 year moving average and standard deviation of the time series. Thus the first order difference time series of $\left(B_{z}, P_{d}\right.$ and $\left.r_{m p}\right)$ is considered as stationary to carry out further analysis and obtain the forecast.

Next we need to determine the ARIMA parameters $p, d$ and $q$ and their seasonal components $\mathrm{P}, \mathrm{D}$ and $\mathrm{Q}$ as defined above. We followed ACF and PACF analysis to get the range of most likely parameters and carry out grid search. Based on the AIC criterion we then determined the best fit parameters that turns out to be $(1,0,1) \times(0,0,1,12)$ for $B_{z}$ and $(1,1,1) \times(0,0,1,12)$ for $P_{d}$ corresponding to the lowest value of AIC. Using these parameters the forecast for the time series of $B_{z}$ and $P_{d}$ is obtained as shown in Fig.A.1. The solid black line represents the actual monthly averaged values of IMF$B_{z}$ and $P_{d}$ obtained from the OMNI2 data base. The solid red and violet lines are forecast values of IMF- $B_{z}$ and $P_{d}$ obtained using the ARIMA method. We compared the predicted values of $P_{d}$ and IMF- $B_{z}$ by ARIMA method with the observed values of $P_{d}$ and IMF- $B_{z}$ obtained from OMNI2 data base between the period 2018-01-01 and 201903-01. It is clearly evident from the forecast of $P_{d}$ that the predicted and the observed values between the period 2018-01-01 and 2019-03-01 are well in agreement. This is, however, not the case of $B_{z}$ which rather shows a simple linear extrapolation curve without any variations indicating that the IMF- $B_{z}$ does indeed stochastic in nature. 

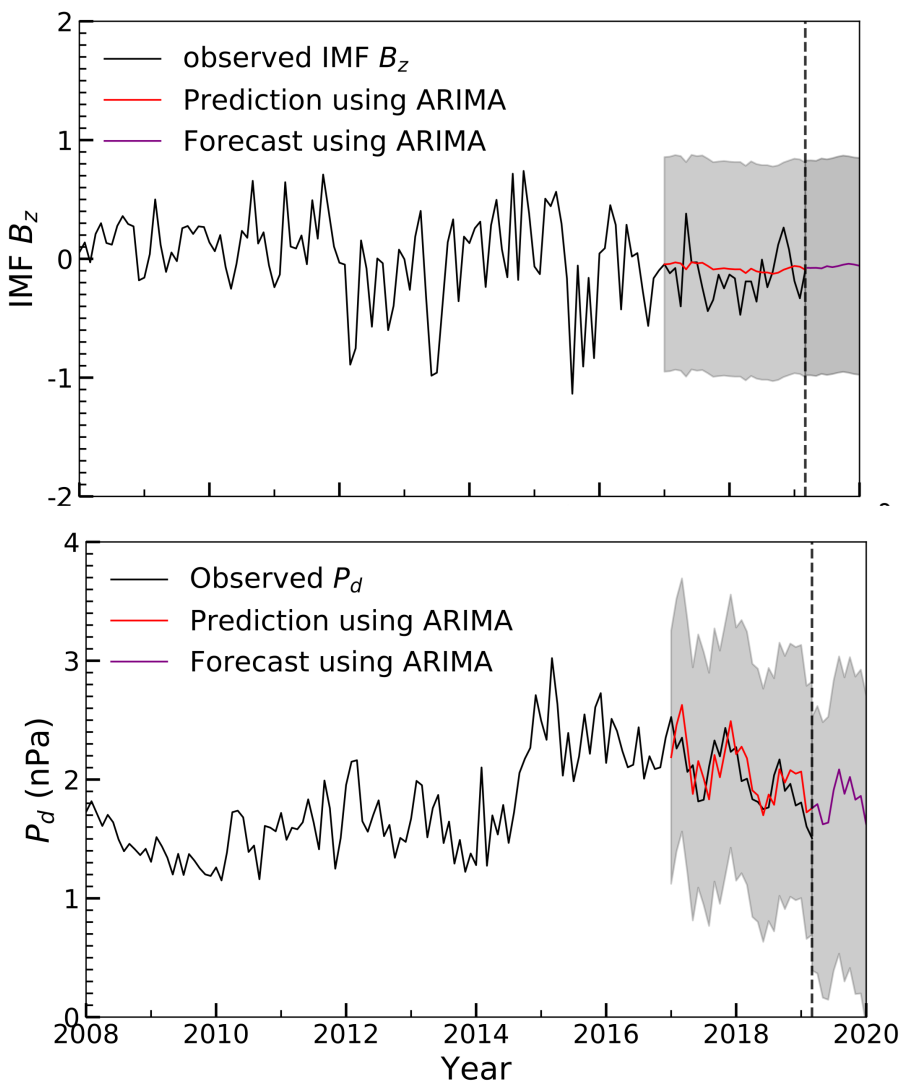

Figure A.1. The forecast of IMF- $B_{z}$ (upper panel) and $P_{d}$ (lower panel). The solid line in black represents the actual monthly averaged values of IMF- $B_{z}$ and $P_{d}$, while the solid red and violet lines are the predicted and forecast values of IMF- $B_{z}$ and $P_{d}$ obtained using the ARIMA method for the period between 2018-01-01 and 2019-03-01 and between 2019-03-02 to 2020-12-31, respectively. The grey band shown is the $95 \%$ confidence interval. The vertical line demarcate the time period from when we have shown the forecast values.

The forecast of $B_{z}$ and $P_{d}$ is then corrected for the first order difference and implemented in the models (L10 and L11) to compute the forecast of $r_{\mathrm{mp}}$ as shown in Fig.A.2. Thereafter, the MP shape for 2020 is obtained as shown in Fig.8. Fig.A.2 shows the forecast of $r_{\mathrm{mp}}$ using the ARIMA method. The solid black line is the calculated $r_{\mathrm{mp}}$ directly using the observed IMF- $B_{z}$ and $P_{d}$ from OMNI2 data base. The solid red and violet lines are predicted values of $r_{\mathrm{mp}}$. We compared the predicted values between 2018-01-01 and 2019-03-01 with the estimated values of $r_{\mathrm{mp}}$ directly from the IMF- $B_{z}$ and $P_{d}$. It is evident from Fig.A.2 that the predicted and the estimated values of $r_{\mathrm{mp}}$ between the period 2018-01-01 and 2019-03-01 are well in agreement. For the prediction of $r_{\mathrm{mp}}$, we have used the predicted non-zero value of IMF- $B_{z}$, as shown Fig.A.1. However, we have carried out the computation by assuming $B_{z}=0$ and found no significant change in $r_{\mathrm{mp}}$ compared with the computations using a finite non-zero value of IMF- $B_{z}$ (for example, for $r_{\mathrm{mp}}$ at 2020, we used $\left.B_{z}=0.038\right)$.

From Fig.A.1 and Fig.A.2, we see that the predicted values of $P_{d}$ and $r_{\mathrm{mp}}$ by ARIMA method agree well with that of the observed values obtained from OMNI2 data base. This verifies the forecast of MP shape using the ARIMA method. 

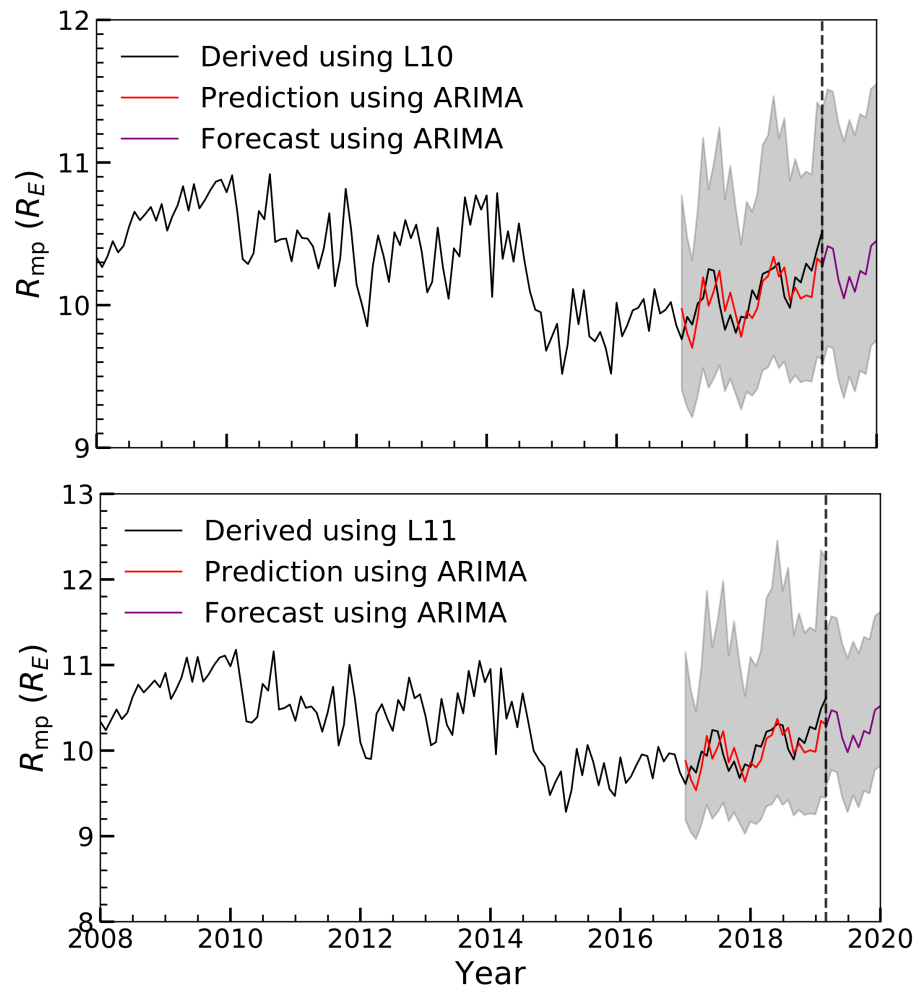

Figure A.2. The forecast of $r_{m} p$. The upper panel uses Lin et al. (2010) (L10) (§3.1.1) and the lower panel uses Lu et al. (2011) (L11) (§3.1.2) models. The solid line in black represents the actual monthly averaged values of $r_{m} p$, while the solid red and violet lines are the predicted and forecast values of $r_{m} p$ obtained using the ARIMA method for the period between 2018-01-01 and 2019-03-01 and between 2019-03-02 to 2020-12-31, respectively. The grey band shown is the $95 \%$ confidence interval. The vertical line demarcate the time period from when we have shown the forecast values.

\section{Acknowledgments}

This work has made use of NASA's OMNIWEB services Data System. The authors thank the free data use policy of the National Solar Observatory (NSO/KP, NSO/SOLIS and NSO/GONG), OMNI2 from NASA and WDC-SILSO at Royal Observatory, Belgium, Brussels. JP acknowledges the ISEE International Collaborative Research Program for support during this work. SKB acknowledges the support by the PIFI (Project No. 2015PM066) program of the Chinese Academy of Sciences and the NSFC (Grants No. 117550110422, 11433006, 11790301, and 11790305).

\section{References}

Ananthakrishnan, S., Balasubramanian, V., \& Janardhan, P. (1995, April). Latitudinal Variation of Solar Wind Velocity. Space Sci. Rev., 72, 229-232. doi: 10 $.1007 / \mathrm{BF} 00768784$

Ananthakrishnan, S., Coles, W. A., \& Kaufman, J. J. (1980, November). Microturbulence in solar wind streams. J. Geophys. Res., 85, 6025-6030. doi: 10.1029/ JA085iA11p06025

Balasubramanian, V., Janardhan, P., Srinivasan, S., \& Ananthakrishnan, S. 
(2003, March). Interplanetary scintillation observations of the solar wind disappearance event of May $1999 . \quad J$. Geophys. Res., 108, 1121-+. doi: 10.1029/2002JA009516

Beard, D. B. (1960).

J. Geophys. Res., 65, 3559-3568. doi: 10.1029/JZ065i011p03559

Bhowmik, P., \& Nandy, D. (2018, December). Prediction of the strength and timing of sunspot cycle 25 reveal decadal-scale space environmental conditions. Nature Communications. doi: 10.1038/s41467-018-07690-0

Bisoi, S. K., Janardhan, P., Chakrabarty, D., Ananthakrishnan, S., \& Divekar, A. (2014, January). Changes in Quasi-periodic Variations of Solar Photospheric Fields: Precursor to the Deep Solar Minimum in Cycle 23? Solar Phys., 289, 41-61. doi: 10.1007/s11207-013-0335-3

Bisoi, S. K., Janardhan, P., Ingale, M., Subramanian, P., Ananthakrishnan, S., Tokumaru, M., \& Fujiki, K. (2014, August). A study of density modulation index in the inner heliospheric solar wind during solar cycle 23. Astrophys. J., 793, 8-15.

Boardsen, S. A., Eastman, T. E., Sotirelis, T., \& Green, J. L. (2000). J. Geophys. Res., 105, 23,19323,219. doi: 10.1029/1998JA000143

Brownlee, J. (2017). Introduction to time series forecasting with python: How to prepare data and develop models to predict the future. Jason Brownlee. Retrieved from https://books.google.co.in/books?id=bA5ItAEACAAJ

Cairns, I. H., \& Grabbe, C. L. (1994, December). Towards an MHD theory for the standoff distance of Earth's bow shock. Geophys. Res. Lett., 21, 2781-2784. doi: 10.1029/94GL02551

Cairns, I. H., \& Lyon, J. G. (1995, September). MHD simulations of Earth's bow shock at low Mach numbers: Standoff distances. J. Geophys. Res., 100, 17173-17180. doi: 10.1029/95JA00993

Cairns, I. H., \& Lyon, J. G. (1996). Magnetic field orientation effects on the standoff distance of Earth's bow shock. Geophys. Res. Lett., 23, 2883-2886. doi: 10 $.1029 / 96 \mathrm{GL} 02755$

Cameron, R. H., Jiang, J., \& Schüssler, M. (2016, June). Solar Cycle 25: Another Moderate Cycle? Astrophys. J. Lett., 823, L22. doi: 10.3847/2041-8205/823/ 2/L22

Chapman, J. F., \& Cairns, I. H. （2003, May). Three-dimensional modeling of Earth's bow shock: Shock shape as a function of Alfvén Mach num-

ber. Journal of Geophysical Research (Space Physics), 108, $1174 . \quad$ doi: 10.1029/2002JA009569

Chapman, S., \& Ferraro, V. C. A. (1931). A new theory of magnetic storms. Terrestrial Magnetism and Atmospheric Electricity. Terr. Magn. Atmos. Electr., 36, 77-97. doi: 10.1029/TE036i002p00077

de Toma, G. (2011, December). Evolution of Coronal Holes and Implications for High-Speed Solar Wind During the Minimum Between Cycles 23 and 24. Solar Phys., 274, 195-217. doi: 10.1007/s11207-010-9677-2

D'Silva, S., \& Choudhuri, A. R. (1993, May). A theoretical model for tilts of bipolar magnetic regions. Astron. Astrophys., 272, 621.

Elsen, R. K., \& Winglee, R. M. (1997, March). The average shape of the Magnetopause: A comparison of three-dimensional global MHD and empirical models. J. Geophys. Res., 102, 4799-4820. doi: 10.1029/96JA03518

Fairfield, D. H. (1971). Average and unusual locations of the Earth's magnetopause and bow shock. J. Geophys. Res., 76, 6700. doi: 10.1029/JA076i028p06700

Fairfield, D. H., Cairns, I. H., Desch, M. D., Szabo, A., Lazarus, A. J., \& Aellig, M. R. (2001, November). The location of low Mach number bow shocks at Earth. J. Geophys. Res., 106, 25361-25376. doi: 10.1029/2000JA000252

Farris, M. H., \& Russell, C. T. (1994, September). Determining the standoff distance of the bow shock: Mach number dependence and use of models. J. Geo- 
phys. Res., 99, 17. doi: 10.1029/94JA01020

Formisano, V. (1979, December). The three-dimensional shape of the bow shock. Nuovo Cimento C Geophysics Space Physics C, 2, 681-692. doi: $10.1007 / \mathrm{BF} 02558125$

Fujiki, K., Tokumaru, M., Hayashi, K., Satonaka, D., \& Hakamada, K. (2016, August). Long-term Trend of Solar Coronal Hole Distribution from 1975 to 2014. Astrophys. J. Lett., 827, L41. doi: 10.3847/2041-8205/827/2/L41

GarcíA, K. S., \& Hughes, W. J. (2007, June). Finding the Lyon-Fedder-Mobarry magnetopause: A statistical perspective. Journal of Geophysical Research (Space Physics), 112, A06229. doi: 10.1029/2006JA012039

Gopalswamy, N., Yashiro, S., \& Akiyama, S. (2016, May). Unusual Polar Conditions in Solar Cycle 24 and Their Implications for Cycle 25. Astrophys. J. Lett., 823, L15. doi: 10.3847/2041-8205/823/1/L15

Gopalswamy, N., Yashiro, S., Mäkelä, P., Michalek, G., Shibasaki, K., \& Hathaway, D. H. (2012, May). Behavior of Solar Cycles 23 and 24 Revealed by Microwave Observations. Astrophys. J. Lett., 750, L42. doi: 10.1088/2041-8205/750/2/ L42

Janardhan, P., \& Alurkar, S. K. (1993, March). Angular source size measurements and interstellar scattering at $103 \mathrm{MHz}$ using interplanetary scintillation. Astron. Astrophys., 269, 119-127.

Janardhan, P., Bisoi, S. K., Ananthakrishnan, S., Tokumaru, M., \& Fujiki, K. (2011, October). The prelude to the deep minimum between solar cycles 23 and 24: Interplanetary scintillation signatures in the inner heliosphere. Geophys. Res. Lett., 38, L20108. doi: 10.1029/2011GL049227

Janardhan, P., Bisoi, S. K., Ananthakrishnan, S., Tokumaru, M., Fujiki, K., Jose, L., \& Sridharan, R. (2015, July). $\quad$ A 20 year decline in solar photospheric magnetic fields: Inner-heliospheric signatures and possible implications.

Journal of Geophysical Research (Space Physics), 120, 5306-5317. doi: 10.1002/2015JA021123

Janardhan, P., Bisoi, S. K., \& Gosain, S. (2010, December). Solar Polar Fields During Cycles 21 - 23: Correlation with Meridional Flows. Solar Phys., 267, 267277. doi: $10.1007 / \mathrm{s} 11207-010-9653-\mathrm{x}$

Janardhan, P., Fujiki, K., Ingale, M., Bisoi, S. K., \& Rout, D. (2018, October). Solar cycle 24: An unusual polar field reversal. Astron. Astrophys., 618, A148. doi: $10.1051 / 0004-6361 / 201832981$

Janardhan, P., Fujiki, K., Kojima, M., Tokumaru, M., \& Hakamada, K. (2005, August). Resolving the enigmatic solar wind disappearance event of 11 May 1999. J. Geophys. Res., 110, 8101-+. doi: 10.1029/2004JA010535

Janardhan, P., Fujiki, K., Sawant, H. S., Kojima, M., Hakamada, K., \& Krishnan, R. (2008, March). Source regions of solar wind disappearance events. $J$. Geophys. Res., 113, 3102-+. doi: 10.1029/2007JA012608

Janardhan, P., Tripathi, D., \& Mason, H. E. (2008, September). The solar wind disappearance event of 11 May 1999: source region evolution. Astron. \& Astrophys. Lett., 488, L1-L4. doi: 10.1051/0004-6361:200809667

Jelínek, K., Němeček, Z., \& Šafránková, J. (2012, May). A new approach to magnetopause and bow shock modeling based on automated region identification. Journal of Geophysical Research (Space Physics), 117, A05208. doi: 10.1029/2011JA017252

Jian, L. K., Russell, C. T., \& Luhmann, J. G. ～(2011, December). Comparing Solar Minimum 23/24 with Historical Solar Wind Records at 1 AU. Solar Phys., 274, 321-344. doi: 10.1007/s11207-011-9737-2

Lin, R. L., Zhang, X. X., Liu, S. Q., Wang, Y. L., \& Gong, J. C. (2010, April). A three-dimensional asymmetric magnetopause model. Journal of Geophysical Research (Space Physics), 115, A04207. doi: 10.1029/2009JA014235 
Lu, J. Y., Liu, Z.-Q., Kabin, K., Zhao, M. X., Liu, D. D., Zhou, Q., \& Xiao, Y. (2011, September). Three dimensional shape of the magnetopause: Global MHD results. Journal of Geophysical Research (Space Physics), 116, A09237. doi: 10.1029/2010JA016418

McComas, D. J., Angold, N., Elliott, H. A., Livadiotis, G., Schwadron, N. A., Skoug, R. M., \& Smith, C. W. (2013, December). Weakest Solar Wind of the Space Age and the Current "Mini" Solar Maximum. Astrophys. J., 779, 2. doi: 10.1088/0004-637X/779/1/2

McComas, D. J., Elliott, H. A., Schwadron, N. A., Gosling, J. T., Skoug, R. M., \& Goldstein, B. E. (2003, May). The three-dimensional solar wind around solar maximum. Geophys. Res. Lett., 30(10), 1517. doi: 10.1029/2003GL017136

Mead, G. D., \& Beard, D. B. (1964). Shape of the Geomagnetic Field Solar Wind Boundary. J. Geophys. Res., 69, 11691179. doi: 10.1029/JZ069i007p01169

Muñoz-Jaramillo, A., Sheeley, N. R., Zhang, J., \& DeLuca, E. E. ～(2012, July). Calibrating 100 Years of Polar Faculae Measurements: Implications for the Evolution of the Heliospheric Magnetic Field. Astrophys. J., 753, 146. doi: 10.1088/0004-637X/753/2/146

Nemecek, Z., \& Safrankova, J. (1991, December). The earth's bow shock and magnetopause position as a result of the solar wind-magnetosphere interaction. Journal of Atmospheric and Terrestrial Physics, 53, 1049-1054. doi: 10.1016/0021 $-9169(91) 90051-8$

Olson, W. P. (1969). The shape of the tilted magnetopause. J. Geophys. Res., 74, 5642. doi: $10.1029 / J A 074 i 024 p 05642$

Peredo, M., Slavin, J. A., Mazur, E., \& Curtis, S. A. ～(1995, May). Threedimensional position and shape of the bow shock and their variation with Alfvenic, sonic and magnetosonic Mach numbers and interplanetary magnetic field orientation. J. Geophys. Res., 100, 7907-7916. doi: 10.1029/94JA02545

Petrinec, S. M., \& Russell, C. T. (1996, January). Near-Earth magnetotail shape and size as determined from the magnetopause flaring angle. J. Geophys. Res., 101, 137-152. doi: 10.1029/95JA02834

Readhead, A. C. S., \& Hewish, A. (1972, April). Galactic Structure and the Apparent Size of Radio Sources. Nature, 236, 440-443. doi: 10.1038/236440a0

Richardson, J. D., Wang, C., \& Paularena, K. I. (2001). The solar wind: from solar minimum to solar maximum. Advances in Space Research, 27, 471-479. doi: 10 .1016/S0273-1177(01)00074-6

Riley, P., Lionello, R., Linker, J. A., Cliver, E., Balogh, A., Beer, J., .. Koutchmy, S. (2015, April). Inferring the Structure of the Solar Corona and Inner Heliosphere During the Maunder Minimum Using Global Thermodynamic Magnetohydrodynamic Simulations. Astrophys. J., 802, 105. doi: 10.1088/0004-637X/802/2/105

Samsonov, A. A., Bogdanova, Y. V., Branduardi-Raymont, G., Safrankova, J., Nemecek, Z., \& Park, J.-S. (2019). Long-term variations in solar wind parameters, magnetopause location, and geomagnetic activity over the last five solar cycles. Journal of Geophysical Research: Space Physics, O(0), 0. doi: 10.1029/2018JA026355

Sánchez-Sesma, J. (2016, July). Evidence of cosmic recurrent and lagged millenniascale patterns and consequent forecasts: multi-scale responses of solar activity (SA) to planetary gravitational forcing (PGF). Earth System Dynamics, 7, 583-595. doi: 10.5194/esd-7-583-2016

Schatten, K. H., \& Pesnell, W. D. (1993, October). An early solar dynamo prediction: Cycle 23 is approximately cycle 22 . Geophys. Res. Lett., 20, 2275-2278. doi: 10.1029/93GL02431

Shue, J.-H., Chao, J. K., Fu, H. C., Russell, C. T., Song, P., Khurana, K. K., \& Singer, H. J. (1997, May). A new functional form to study the solar wind control of the magnetopause size and shape. J. Geophys. Res., 102, 9497-9512. 
doi: 10.1029/97JA00196

Shue, J.-H., Song, P., Russell, C. T., Steinberg, J. T., Chao, J. K., Zastenker, G., ... Kawano, H. (1998, August). Magnetopause location under extreme solar wind conditions. J. Geophys. Res., 103, 17691-17700. doi: 10.1029/98JA01103

Shukhtina, M. A., \& Gordeev, E. (2015). In situ magnetotail magnetic flux calculation. Annales Geophysicae, 33(6), 769-781. Retrieved from http://www . ann -geophys.net/33/769/2015/ doi: 10.5194/angeo-33-769-2015

Sibeck, D. G., Lopez, R. E., \& Roelof, E. C. (1991, April). Solar wind control of the magnetopause shape, location, and motion. J. Geophys. Res., 96, 5489-5495. doi: 10.1029/90JA02464

Spreiter, J. R., \& Briggs, B. R. (1962, January). Theoretical Determination of the Form of the Boundary of the Solar Corpuscular Strea Produced by Interaction with the Magnetic Dipole Field of the Earth. J. Geophys. Res., 67, 37-51. doi: 10.1029/JZ067i001p00037

Sun, X., Hoeksema, J. T., Liu, Y., \& Zhao, J. (2015, January). On Polar Magnetic Field Reversal and Surface Flux Transport During Solar Cycle 24. Astrophys. J., 798, 114. doi: 10.1088/0004-637X/798/2/114

Tóth, G., Sokolov, I. V., Gombosi, T. I., Chesney, D. R., Clauer, C. R., De Zeeuw, D. L., ... Kóta, J. (2005, December). Space Weather Modeling Framework: A new tool for the space science community. J. Geophys. Res., 110, 12226-+. doi: 10.1029/2005JA011126

Upton, L. A., \& Hathaway, D. H. (2018, August). An Updated Solar Cycle 25 Prediction With AFT: The Modern Minimum. Geophys. Res. Lett., 45, 8091-8095. doi: 10.1029/2018GL078387

Wang, Y., Sibeck, D. G., Merka, J., Boardsen, S. A., Karimabadi, H., Sipes, T. B., ... Lin, R. (2013, May). A new three-dimensional magnetopause model with a support vector regression machine and a large database of multiple spacecraft observations. Journal of Geophysical Research (Space Physics), 118, 2173-2184. doi: 10.1002/jgra.50226

Yang, Y.-H., Chao, J. K., Dmitriev, A. V., Lin, C.-H., \& Ober, D. M.

$(2003$ March). Saturation of IMF $\mathrm{B}_{z}$ influence on the position of dayside magnetopause. Journal of Geophysical Research (Space Physics), 108, $1104 . \quad$ doi: 10.1029/2002JA009621

Zachilas, L., \& Gkana, A. (2015, May). On the Verge of a Grand Solar Minimum: A Second Maunder Minimum? Solar Phys., 290, 1457-1477. doi: 10.1007/s11207 -015-0684-1

Zhigulevsk, V. N., \& Romishevskii, E. A. (1959). Soviet Phys. Doklady, 5, 1001-1004.

Zhong, J., Wan, W. X., Wei, Y., Fu, S. Y., Jiao, W. X., Rong, Z. J., ... Han, X. H. (2014, December). Increasing exposure of geosynchronous orbit in solar wind due to decay of Earth's dipole field. Journal of Geophysical Research (Space Physics), 119, 9816-9822. doi: 10.1002/2014JA020549

Zolotova, N. V., \& Ponyavin, D. I. (2014, May). Is the new Grand minimum in progress? Journal of Geophysical Research (Space Physics), 119, 3281-3285. doi: 10.1002/2013JA019751 